\title{
A CLASSIFICATION OF PERIODIC TIME-DEPENDENT GENERALIZED HARMONIC OSCILLATORS USING A HAMILTONIAN ACTION OF THE SCHRÖDINGER-VIRASORO GROUP
}

\author{
JEREMIE UNTERBERGER \\ Département de Mathématiques, \\ Université Henri Poincaré Nancy I, BP 239, \\ 54506 Vandoeuvre-les-Nancy Cedex, France \\ Received 4 December 2009 \\ Revised 7 January 2010
}

\begin{abstract}
In the wake of a preceding article [31] introducing the Schrödinger-Virasoro group, we study its affine action on a space of $(1+1)$-dimensional Schrödinger operators with time- and space-dependent potential $V$ periodic in time. We focus on the subspace corresponding to potentials that are at most quadratic in the space coordinate, which is in some sense the natural quantization of the space of Hill (Sturm-Liouville) operators on the one-dimensional torus. The orbits in this subspace have finite codimension, and their classification by studying the stabilizers can be obtained by extending Kirillov's results on the orbits of the space of Hill operators under the Virasoro group. We then explain the connection to the theory of Ermakov-Lewis invariants for time-dependent harmonic oscillators. These exact adiabatic invariants behave covariantly under the action of the Schrödinger-Virasoro group, which allows a natural classification of the orbits in terms of a monodromy operator on $L^{2}(\mathbb{R})$ which is closely related to the monodromy matrix for the corresponding Hill operator.
\end{abstract}

Keywords: Time-dependent Schrödinger equation; harmonic oscillators; Hill operators; Virasoro algebra; Schrödinger-Virasoro algebra; Ermakov-Lewis invariants; monodromy; adiabatic theorem; representation theory of infinite-dimensional Lie algebras.

AMS Subject Classification: 22E65, 22E70, 34A26, 34L40, 35Q40

\section{Introduction}

The Schrödinger-Virasoro Lie algebra $\mathfrak{s v}$ was originally introduced in Henkel [18] as a natural infinite-dimensional extension of the Schrödinger algebra. Recall that the latter is defined as the algebra of projective Lie symmetries of the free Schrödinger equation in $(1+1)$ dimensions

$$
\left(-2 i \partial_{t}-\partial_{r}^{2}\right) \psi(t, r)=0 .
$$


These act on Eq. (0.1) as the following first-order operators

$$
\begin{aligned}
L_{n} & =-t^{n+1} \partial_{t}-\frac{1}{2}(n+1) t^{n} r \partial_{r}+\frac{i}{4}(n+1) n t^{n-1} r^{2}-(n+1) \lambda t^{n}, \\
Y_{m} & =-t^{m+\frac{1}{2}} \partial_{r}+i\left(m+\frac{1}{2}\right) t^{m-\frac{1}{2}} r \\
M_{p} & =i t^{p}
\end{aligned}
$$

with $\lambda=1 / 4$ and $n=0, \pm 1, m= \pm \frac{1}{2}, p=0$. The zeroth-order terms in $(0.2)$ correspond at the level of the group to the multiplication of the wave function by a phase. To be explicit, the six-dimensional Schrödinger group $\mathcal{S}$ acts on $\psi$ by the following transformations

$$
\left(L_{-1}, L_{0}, L_{1}\right): \psi(t, r) \rightarrow \psi^{\prime}\left(t^{\prime}, r^{\prime}\right)=(c t+d)^{-1 / 2} e^{-\frac{1}{2} i c r^{2} /(c t+d)} \psi(t, r),
$$

where $t^{\prime}=\frac{a t+b}{c t+d}, r^{\prime}=\frac{r}{c t+d}$ with $a d-b c=1$;

$$
\left(Y_{ \pm \frac{1}{2}}\right): \psi(t, r) \rightarrow \psi\left(t, r^{\prime}\right)=e^{-i\left(\left(v t+r_{0}\right)(r-v / 2)\right.} \psi(t, r)
$$

where $r^{\prime}=r-v t-r_{0}$;

$$
\left(M_{0}\right): \psi(t, r) \rightarrow e^{-i \gamma} \psi(t, r) .
$$

Altogether these transformations make up a group $\mathcal{S}$, called Schrödinger group, which is isomorphic to a semi-direct product of $\mathrm{SL}(2, \mathbb{R})$ (corresponding to timereparametrizations $(0.3)$ ) by a Heisenberg group $\mathcal{H}_{1}$ (corresponding to the Galilei transformations $(0.4),(0.5))$. Note that the last transformation (0.5) (multiplication by a constant phase) is generated by the commutators of the Galilei transformations (0.4) - these do not commute because of the added phase terms, which produce a central extension.

Now $\mathfrak{s v} \simeq\left\langle L_{n}, Y_{m}, M_{p} \mid n, p \in \mathbb{Z}, m \in \frac{1}{2}+\mathbb{Z}\right\rangle$ made up of all linear combinations of the generators corresponding to all possible integer or half-integer indices - is a Lie algebra, as can be checked by direct computation. Similarly to the Lie algebra of the Schrödinger group, it is a semi-direct product, $\mathfrak{s v} \simeq \mathfrak{g}_{0} \ltimes \mathfrak{h}$, where $\mathfrak{g}_{0}=\left\langle L_{n}\right\rangle_{n \in \mathbb{Z}}$ is the centerless Virasoro algebra and $\left.\mathfrak{h}=\left\langle Y_{m}, M_{p}\right| m \in \frac{1}{2}+\mathbb{Z}, p \in \mathbb{Z}\right\}$ is a two-step nilpotent infinite-dimensional Lie algebra which extends the Heisenberg Lie algebra. It may be exponentiated into a group (the Schrödinger-Virasoro group) that we denote by SV. The paper [31], by Roger and the author, studies this Lie algebra for its own sake from different points of view, including representation theory, deformations, central extensions. There is a hope that this Lie algebra or related ones may help classify strongly anisotropic critical systems and models pertaining to out-of-equilibrium statistical physics, notably ageing phenomena, for which the anisotropic dilation $(t, r) \rightarrow\left(\lambda^{2} t, \lambda r\right)(\lambda \in \mathbb{R})$ holds. A systematic investigation of the consequences of a symmetry of the physical system under consideration under the Schrödinger group or related groups has been conducted since the mid-90s (for a short survey, see [20]). 
The starting point for this work is a little different. One of the possible motivations for introducing this Lie algebra in the first place is that the group of Lie symmetries of any Schrödinger operator $-2 i \partial_{t}-\partial_{r}^{2}+V(t, r)$ may be represented as a linear combination of the generators introduced in (0.2). In other words, for any particular Schrödinger operator, the Lie algebra of symmetries is finite-dimensional, but the symmetry algebras of all Schrödinger operators are contained in $\mathfrak{s} \mathfrak{v}$ in the above realization (see Sec. 2.5 below for a more precise statement). The proof lies in some sense in a classical paper by Niederer (see [28]) — who never considered the algebra generated by all possible symmetries.

Another related way to look at it is that $\mathfrak{s v}$ acts on the space of Schrödinger operators. More precisely, SV acts on the affine space of Schrödinger operators with time- and space-dependent potential at most quadratic in the space coordinate. We call it $\mathcal{S}_{\leq 2}^{\text {aff }}:=\left\{-2 i \partial_{t}-\partial_{r}^{2}+V_{2}(t) r^{2}+V_{1}(t) r+V_{0}(t)\right\}$. It is assumed that $V_{0}, V_{1}$ and $V_{2}$ are $2 \pi$-periodic in time; this hypothesis is natural when one sets $t=e^{i \theta}$ $(\theta \in \mathbb{R} / 2 \pi \mathbb{Z})$ as a coordinate on the unit circle, so that the generator $L_{n}$ acts as $-e^{i n \theta} \partial_{\theta}+\cdots$. This restricted space is in some sense minimal, which can be seen from the fact that $\mathcal{S}_{\leq 2}^{\text {aff }}$ may be expressed in terms of three functions of time, just like the elements of $\mathfrak{s v}$. The phase terms in (0.2) add by commutation with the free Schrödinger equation terms of order $1, r$ and $r^{2}$. One can show that the orbit of any Schrödinger operator $D \in \mathcal{S}_{\leq 2}^{\text {aff }}$ has finite-codimension in this space. Hence this space appears to be natural from a representation point of view.

In Sec. 2 below (see Sec. 2.4), we classify the orbits of SV in $\mathcal{S}_{\leq 2}^{\text {aff }}$. The classification is mainly an extension of Kirillov's results on the classification of the orbits of the space of Hill operators under the Virasoro group. These are operators of the type $\partial_{t}^{2}+u(t)$. It is well known (see for instance Guieu [13] or [14]) that the group of orientation-preserving diffeomorphisms Diff $+(\mathbb{R} / 2 \pi \mathbb{Z})$ of the circle which exponentiates the centerless Virasoro algebra - acts on the affine space of Hill operators. Now the remarkable fact (despite the apparent differences between the two problems) is that the action of the Virasoro group Diff $+(\mathbb{R} / 2 \pi \mathbb{Z}) \subset \mathrm{SV}$ on the quadratic part of the potential, $V_{2}(t) r^{2}$, is equivalent to that of Diff $+(\mathbb{R} / 2 \pi \mathbb{Z})$ on the Hill operator $\partial_{t}^{2}+V_{2}(t)$. The reason comes from the fact that the Hill operator is the corresponding classical problem in the semiclassical limit (see Sec. 3.2). Hence part of the classification may be borrowed directly from the work of Kirillov (see [23]). Kirillov obtains his classification by studying the isotropy algebra $\operatorname{Lie}\left(\operatorname{Stab}_{u}\right):=\left\{X \in \operatorname{Lie}\left(\operatorname{Diff}_{+}(\mathbb{R} / 2 \pi \mathbb{Z})\right) \mid X \cdot\left(\partial^{2}+u\right)=0\right\}$. There is another equivalent description in terms of the lifted monodromy, which can be explained briefly as follows. If $\left(\psi_{1}, \psi_{2}\right)$ is a basis of solutions of the ordinary differential equation $\left(\partial_{t}^{2}+u(t)\right) \psi(t)=0$, then (by Floquet's theory)

$$
\left(\begin{array}{l}
\psi_{1}\left(t_{0}+2 \pi\right) \\
\psi_{2}\left(t_{0}+2 \pi\right)
\end{array}\right)=M \cdot\left(\begin{array}{l}
\psi_{1}\left(t_{0}\right) \\
\psi_{2}\left(t_{0}\right)
\end{array}\right),
$$

where $M$ is some matrix (called monodromy matrix) with determinant 1 which does not depend on the base point $t_{0}$. If $M$ is elliptic, i.e. conjugate to a rotation, then the 
eigenvectors for $M$ are multiplied by a phase $e^{i \theta}$. If $M$ is hyperbolic, i.e. conjugate to a Lorentz shift $\left(\begin{array}{c}e^{\lambda} \\ e^{-\lambda}\end{array}\right)$, then the eigenvectors are multiplied by a real factor $e^{ \pm \lambda}$, hence the solutions of the Hill equation are unstable, going either to zero or to infinity when $t \rightarrow \pm \infty$. A nice way to see it (and made rigorous in Sec. 2) is to imagine the vector $\left(\begin{array}{l}\psi_{1}(t) \\ \psi_{2}(t)\end{array}\right)$ as "rotating" in the plane (it may also change norm but never vanishes). The curve described by this vector may be lifted to the Riemann surface of the logarithm for instance (obtained from the cut plane $\mathbb{C} \backslash \mathbb{R}_{-}$), so that it turns by an angle unambiguously defined in $\mathbb{R}$. This gives the lifted monodromy.

The space $\mathcal{S}_{<2}^{\text {aff }}$ has been considered independently by mathematicians and physicists, with similar motivations but different methods (that turn out to be equivalent in the end). The general idea was to solve the evolution problem associated with $D \in \mathcal{S}_{<2}^{\text {aff }}$, i.e. to show that the Cauchy problem $D \psi=0$ with initial condition $\psi(0, r)=\psi_{0}(r)$ has a unique solution and compute it explicitly. The usual method in mathematical physics for such time-dependent problems is to consider the adiabatic approximation: if one puts formally a small coefficient $\varepsilon$ in front of $\partial_{t}$, the problem is equivalent by dilating the time coordinate to the equation $\left(-2 i \partial_{t}-\partial_{r}^{2}+V(\varepsilon t, r)\right) \psi=0$, so that $V$ is a potential that is slowly varying in time. Suppose that $\Delta_{\varepsilon}(t):=-\partial_{r}^{2}+V(\varepsilon t, r)$ has a pure point spectrum $\left\{\lambda_{n}(t), n \in \mathbb{N}\right\}$ for every $t$, where $\lambda_{n}$ is $C^{\infty}$ in $t$, say, and let $\psi_{n}(t)$ be a normalized eigenfunction of $\Delta_{\varepsilon}(t)$ satisfying the gauge-fixing condition $\left\langle\psi_{n}(t), \dot{\psi}_{n}(t)\right\rangle=0$. Then there exists a parallel transport operator $W(s, t)$ carrying the eigenspace with eigenvalue $\lambda_{n}(s)$ to the eigenspace with eigenvalue $\lambda_{n}(t)$, and a phase operator $\Phi(s, t)$, given simply by the multiplication by a phase $e^{\frac{i}{2} \int{ }^{t} \lambda_{n}(s) d s}$ on each eigenspace, such that the solution of the Schrödinger equation is given at first order in $\varepsilon$ by the composition of $W$ and $\Phi$. One may see the solutions formally as flat sections for a connection (called Berry connection) related in simple terms to the phase operator (see [4]). This scheme may be iterated, giving approximate solutions to the Schrödinger equation that are correct to any order in $\varepsilon$ (see for instance Joye [21]), but it is rarely the case that one can give exact solutions. By considering the related classical problem, Hagedorn (see [16]) constructs a set of raising and lowering operators (generalizing those associated to the usual harmonic oscillator) for general Schrödinger operators in $\mathcal{S}_{\leq 2}^{\text {aff }}$, and uses them to solve the equation explicitly. The same set of operators had been considered previously by two quantum physicists, Lewis and Riesenfeld (see [26]), and obtained by looking for an exact invariant, i.e. for a time-dependent operator $I(t)$ (not including the time-derivative) such that $\frac{d I}{d t}=\frac{\partial I}{\partial t}+i\left[I(t), \frac{1}{2}\left(\partial_{r}^{2}-V(t, r)\right)\right]=0$. They find for each operator $D$ in $\mathcal{S}_{\leq 2}^{\text {aff }}$ a family of invariants (sometimes called the Ermakov-Lewis invariants, see [29]) depending on an arbitrary real solution $\xi$ of a certain differential equation of order 3 (see Proposition 3.1.4), constructed out of generalized raising and lowering operators and spectrally equivalent to the standard harmonic oscillator $-\frac{1}{2}\left(\partial_{r}^{2}-r^{2}\right)$. These invariants have been used to solve quite a few physical problems, ranging from quantum mechanics for charged particles to cosmology (see [11, 12, 29, 30] for instance). 
It turns out that very few Schrödinger operators have an exact invariant of the type $I(t)=f_{2}(t, r) \partial_{r}^{2}+f_{1}(t, r) \partial_{r}+f_{0}(t, r)$. These may be expressed, as shown by Lewis and Leach (see [25]), in terms of three arbitrary functions of time (the exact expression is complicated). Exact invariants allow in principle to solve explicitly the original problem, at least if one knows how to diagonalize them (which is the case here). Hence (provided one requires that an exact invariant exists), the space $\mathcal{S}_{\leq 2}^{\text {aff }}$ is maximal.

There are three new features here:

- the action of the Schrödinger-Virasoro group on $\mathcal{S}_{\leq 2}^{\text {aff }}$ (which is essentially a conjugate action, leaving all invariant quantities unchanged, for instance the spectrum and the monodromy) makes it possible to reduce the study to five families of operators, with qualitatively different properties (see Sec. 2.4). They are mainly characterized by the monodromy of the associated Hill operator $\partial_{t}^{2}+V_{2}(t)$, but there also appear some non-generic orbits in cases when the quadratic and linear parts of the potential are "resonant". The non-periodic case is much simpler, since (locally in time) all Schrödinger operators in $\mathcal{S}_{\leq 2}^{\text {aff }}$ are formally equivalent (see Sec. 3 below). The coefficients of the Ermakov-Lewis invariants are related in a very simple way to the invariants of the orbits;

- one is interested in Schrödinger operators with time-periodic potential. Hence one may consider (as in the case of ordinary differential operators, see above) the monodromy, which is a bounded operator acting on $L^{2}(\mathbb{R})$. The monodromy operator is given explicitly and shown to be closely related to the classical monodromy of the related Hill operator;

- the computation of the monodromy in the case when the associated Hill operator is hyperbolic (see above) requires the use of an Ermakov-Lewis invariant associated to a purely imaginary function $\xi$, which is equivalent to the standard harmonic "repulsor" $-\frac{1}{2}\left(\partial_{r}^{2}+r^{2}\right)$. The reason (explained more precisely in Sec. 3 below) is that the usual Ermakov-Lewis invariants are defined only if $I_{V_{2}}(\xi)>0$, where the invariant quantity $I_{V_{2}}(\xi)$ (quadratic in $\xi$ ) is associated to the Hill operator $\partial_{t}^{2}+V_{2}(t)$ and its stabilizer $\xi(t)$ in $\operatorname{Lie}\left(\operatorname{Diff}_{+}(\mathbb{R} / 2 \pi \mathbb{Z})\right)$. The stabilizer satisfies a linear differential equation of order 3 and has generically only one periodic solution (up to a constant). If one does not require $\xi$ to be periodic, then $I_{u}(\xi)$ may be chosen to be positive, which is perfectly suitable for a local study (in time) but is of little practical use for the computation of the monodromy. If, however, one requires that $\xi$ be periodic, then $I_{u}(\xi)$ is negative in the hyperbolic case, unless one chooses $\xi$ to be purely imaginary. Hence one is naturally led to use the spectral decomposition of the harmonic "repulsor" (which has an absolutely continuous spectrum equal to the whole real line). Usually there is no adiabatic scheme, hence no phase operator, in the case when eigenvalues are not separated by a gap. But in this very particular case, such a phase operator may be computed and is very analogous to that obtained in the elliptic case, for which the spectrum is discrete. There also 
exists some non-generic cases (corresponding to a unipotent monodromy matrix for the underlying Hill operator) for which $I_{V_{2}}(\xi)=0$. The natural invariant is then spectrally equivalent either to the bare Laplacian $-\frac{1}{2} \partial_{r}^{2}$ or to the Airy operator $-\frac{1}{2}\left(\partial_{r}^{2}-r\right)$.

One of the main results may be stated as follows (see Secs. 3.4-3.6): the monodromy operator is unitarily equivalent to the unitary multiplication operator $f(k) \rightarrow e^{i k T-i \pi \gamma} f(k)$, where $\gamma$ is some constant and $k$ is the spectral parameter of the model operator $-\frac{1}{2}\left(\partial_{r}^{2}+\kappa r^{2}\right)(\kappa= \pm 1,0)$ or $-\frac{1}{2}\left(\partial_{r}^{2}-r\right)$, and $T=\int_{0}^{2 \pi} \frac{d u}{\xi(u)}$ ( $\xi$ real) or $i \int_{0}^{2 \pi} \frac{d u}{\xi(u)}$ ( $\xi$ imaginary). The above integrals must be understood in a generalized sense if $\xi$ has some zeros; a complex deformation of contour is needed then. Comparing with the usual Berry phase $e^{\frac{i}{2} \int_{0}^{2 \pi} \lambda_{k}(s) d s}$, one sees that the eigenvalue $\lambda_{k}(t)=-2 k$ is a constant, but that the natural (possibly singular) time-scale is $\tau:=\int^{t} \frac{d u}{\xi(u)}$.

The set of all possible monodromy operators has been determined in [17] by remarking that, for quadratic (time-dependent) Hamiltonians, the unitary propagator is determined up to a time-dependent phase by the classical flow obtained by letting $\hbar \rightarrow 0$. This makes it possible to deduce the quantum monodromy operator up to a phase from the classical monodromy matrix. However, these results do not allow to determine the phase, which we are able to do by giving a family of normal forms for elements in $\mathcal{S}_{\leq 2}^{\text {aff }}$. A great part of the interest of the present work is the explicit connection between the orbit data obtained by algebraic tools, and the evolution operator obtained through the Ermakov-Lewis method out of the spectral decomposition of the model operators $-\frac{1}{2}\left(\partial_{r}^{2} \pm r^{2}\right),-\frac{1}{2} \partial_{r}^{2},-\frac{1}{2}\left(\partial_{r}^{2}-r\right)$.

The paper is organized as follows.

Section 1 is preliminary and contains notations and results (contained in [31]) concerning the Schrödinger-Virasoro group and its action on Schrödinger operators.

Section 2 is dedicated to the classification of the orbits and of the isotropy subgroups $G_{D}:=\{g \in \mathrm{SV} \mid g . D=D\}, D \in \mathcal{S}_{\leq 2}^{\text {aff }}$ (see Sec. 2.4). It contains long but necessary preliminaries on the action of the Virasoro group on Hill operators. The connection to the results of Niederer is made in the last paragraph.

We solve the monodromy problem for the Schrödinger operators of the form $-2 i \partial_{t}-\partial_{r}^{2}+V_{2}(t) r^{2}+\gamma(\gamma$ constant $)$ in Sec. 3. We study first the corresponding classical problem given by the associated Hill operator, $\ddot{x}+V_{2}(t) x=0$ (an ordinary differential equation). The solution of the quantum problem is then easily deduced from that of the classical problem. In either case, the monodromy is obtained by relating the Ermakov-Lewis invariants to the orbit data.

Finally, we show in Sec. 4 how to parametrize a general Schrödinger operator $-2 i \partial_{t}-\partial_{r}^{2}+V_{2}(t) r^{2}+V_{1}(t) r+V_{0}(t) \in \mathcal{S}_{\leq 2}^{\text {aff }}$ by means of a three-dimensional invariant $\left(\xi(t), \delta_{1}(t), \delta_{2}(t)\right)$ (see Definition 4.2). The parametrization is one-to-one or "almost" one-to-one depending on the orbit class of the potential $V_{2}$ (SV-orbits in $\mathcal{S}_{\leq 2}^{\text {aff }}$ have generically codimension 2 , whereas adjoint orbits corresponding to the invariant have generically codimension 2 or 3 ). The action of the Schrödinger-Virasoro 
group on $\mathcal{S}_{\leq 2}^{\text {aff }}$, once written in terms of the invariant, becomes much simpler, and is easily shown to be Hamiltonian for a natural symplectic structure. A generalized Ermakov-Lewis invariant may also be written in terms of this threedimensional invariant. We then solve the monodromy cases for the "resonant" cases left from Sec. 3.

Notation. The notation $\operatorname{Vect}\left(S^{1}\right):=\left\{\xi(t) \partial_{t} \mid \xi \in \mathbb{C}^{\infty}(\mathbb{R} / 2 \pi \mathbb{Z})\right\}$ will be used for the Lie algebra of $C^{\infty}$-vector fields on the torus $\mathbb{R} / 2 \pi \mathbb{Z}$. The infinite-dimensional group Diff $+(\mathbb{R} / 2 \pi \mathbb{Z})$ of orientation-preserving diffeomorphisms of the torus $\mathbb{R} / 2 \pi \mathbb{Z}$ (also called centerless Virasoro group) has a Lie structure, and its Lie algebra is $\operatorname{Vect}\left(S^{1}\right)$ (see [14] for details).

Let us gather here (for the convenience of the reader) a few notations scattered in the text. Time and space coordinates are usually (at least starting from Sec. 2) denoted by $\theta$ and $x$ (see explanations before Lemma 1.6 for the passage to Laurent coordinates $(t, r))$. Stabilizers in $\operatorname{Vect}\left(S^{1}\right)$ of the Hill operator $\partial_{\theta}^{2}+V_{2}(\theta)$ are usually denoted by $\xi$ (which is either real or purely imaginary). If $\xi$ is purely imaginary, then one sets $\xi:=i \eta$. As for (operator) invariants of the Schrödinger operators (see Sec. 4), we write them as $\frac{1}{2}\left[a(\theta) x^{2}-b(\theta) \partial_{x}^{2}-i c(\theta)\left(x \partial_{x}+\right.\right.$ $\left.\left.\partial_{x} x\right)+d(\theta)\left(-i \partial_{x}\right)+e(\theta) x+f(\theta)\right]$. The correspondence between the vector invariant $\left(\xi, \delta_{1}, \delta_{2}\right)$ and the operator (generalized Ermakov-Lewis) invariant is given in Theorem 4.4 .

\section{The Schrödinger-Virasoro Group and Its Action on Schrödinger Operators}

We recall in this preliminary section the properties of the Schrödinger group proved in [31] that will be needed throughout the paper.

Definition 1.1. (see [31], Definition 1.2) We denote by $\mathfrak{s} \mathfrak{v}(\kappa), \kappa=0$ or $\frac{1}{2}$, the Lie algebra with generators $L_{n}, Y_{m}, M_{n}(n \in \mathbb{Z}, m \in \kappa+\mathbb{Z})$ and the following relations (where $n, p \in \mathbb{Z}, m, m^{\prime} \in \kappa+\mathbb{Z}$ ):

$$
\begin{gathered}
{\left[L_{n}, L_{p}\right]=(n-p) L_{n+p}} \\
{\left[L_{n}, Y_{m}\right]=\left(\frac{n}{2}-m\right) Y_{n+m}, \quad\left[L_{n}, M_{p}\right]=-p M_{n+p}} \\
{\left[Y_{m}, Y_{m^{\prime}}\right]=\left(m-m^{\prime}\right) M_{m+m^{\prime}}} \\
{\left[Y_{m}, M_{p}\right]=0, \quad\left[M_{n}, M_{p}\right]=0 .}
\end{gathered}
$$

If $f$ (resp. $g, h$ ) is a Laurent series, $f=\sum_{n \in \mathbb{Z}} f_{n} t^{n+1}$, resp. $g=\sum_{n \in \kappa+\mathbb{Z}} g_{n} t^{n+\frac{1}{2}}$, $h=\sum_{n \in \mathbb{Z}} h_{n} t^{n}$, then we shall write

$$
\mathcal{L}_{f}=\sum f_{n} L_{n}, \quad \mathcal{Y}_{g}=\sum g_{n} Y_{n}, \quad \mathcal{M}_{h}=\sum h_{n} M_{n}
$$

Note the shift in the indices in the Laurent series which disappears in the Fourier coordinates, see remarks preceding Lemma 1.6 below. 
It is often unimportant (or a matter of taste) in this paper whether the shift index $\kappa$ is 0 or $\frac{1}{2}$ (see remarks after Theorem 2.4.2 though). In this section (unless otherwise stated) $\mathfrak{s v}$ stands indifferently for $\mathfrak{s} \mathfrak{v}(0)$ or $\mathfrak{s v}(1 / 2)$. In the following sections, we shall abbreviate $\mathfrak{s v}(0)$ to $\mathfrak{s v}$ for convenience.

Definition 1.2. (see [31], Definition 1.3) Denote by $d \pi_{\lambda}$ the representation of $\mathfrak{s v}$ as differential operators of order one on $\mathbb{R}^{2}$ with coordinates $t, r$ defined by

$$
\begin{aligned}
& d \pi_{\lambda}\left(L_{n}\right)=-t^{n+1} \partial_{t}-\frac{1}{2}(n+1) t^{n} r \partial_{r}+\frac{1}{4} i(n+1) n t^{n-1} r^{2}-(n+1) \lambda t^{n}, \\
& d \pi_{\lambda}\left(Y_{m}\right)=-t^{m+\frac{1}{2}} \partial_{r}+i\left(m+\frac{1}{2}\right) t^{m-\frac{1}{2}} r \\
& d \pi_{\lambda}\left(M_{p}\right)=i t^{p} .
\end{aligned}
$$

Proposition 1.3. (see [31], Theorem 1.1) (1) The Lie algebra $\mathfrak{s v}$ can be exponentiated to a Lie group denoted by $S V$. It is isomorphic to a semi-direct product $\mathrm{SV}=G_{0} \ltimes H$, where $G_{0} \simeq \operatorname{Diff}_{+}(\mathbb{R} / 2 \pi \mathbb{Z})$ is the group of orientation-preserving diffeomorphisms of the torus $\mathbb{R} / 2 \pi \mathbb{Z}$, and $H \simeq C^{\infty}(\mathbb{R} / 2 \pi \mathbb{Z}) \times C^{\infty}(\mathbb{R} / 2 \pi \mathbb{Z}$ ) (as a vector space) is the product of two copies of the space of infinitely differentiable functions on the circle, with its group structure modified as follows:

$$
\left(\alpha_{2}, \beta_{2}\right) \cdot\left(\alpha_{1}, \beta_{1}\right)=\left(\alpha_{1}+\alpha_{2}, \beta_{1}+\beta_{2}+\frac{1}{2}\left(\alpha_{1}^{\prime} \alpha_{2}-\alpha_{1} \alpha_{2}^{\prime}\right)\right) .
$$

The semi-direct product is given by:

$$
(1 ;(\alpha, \beta)) \cdot(\phi ; 0)=(\phi ;(\alpha, \beta))
$$

and

$$
(\phi ; 0) \cdot(1 ;(\alpha, \beta))=\left(\phi ;\left(\left(\phi^{\prime}\right)^{1 / 2}(\alpha \circ \phi), \beta \circ \phi\right)\right) .
$$

(2) The infinitesimal representation $d \pi_{\lambda}$ of $\mathfrak{s v}$ can be exponentiated to the following representation of the group $\mathrm{SV}$ on $C^{\infty}$ functions of two variables,

(a)

$$
\left(\pi_{\lambda}(\phi ; 0) f\right)\left(t^{\prime}, r^{\prime}\right)=\left(\phi^{\prime}(t)\right)^{-\lambda} e^{\frac{1}{4} i \frac{\phi^{\prime \prime}(t)}{\phi^{\prime}(t)} r^{2}} f(t, r)
$$

if $\phi \in \operatorname{Diff}_{+}(\mathbb{R} / 2 \pi \mathbb{Z})$ induces the coordinate change $(t, r) \rightarrow\left(t^{\prime}, r^{\prime}\right)=$ $\left(\phi(t), r \sqrt{\phi^{\prime}(t)}\right)$;

(b)

$$
\left(\pi_{\lambda}(1 ;(\alpha, \beta)) f\right)\left(t^{\prime}, r^{\prime}\right)=e^{-i\left(\alpha^{\prime}(t) r-\frac{1}{2} \alpha(t) \alpha^{\prime}(t)+\beta(t)\right)} f(t, r)
$$

if $(\alpha, \beta) \in C^{\infty}(\mathbb{R} / 2 \pi \mathbb{Z}) \times C^{\infty}(\mathbb{R} / 2 \pi \mathbb{Z})$ induces the coordinate change $(t, r) \rightarrow$ $\left(t, r^{\prime}\right)=(t, r-\alpha(t))$. 
Definition 1.4. (see [31], Definition 2.1) Let $\mathcal{S}^{\text {lin }}$ be the vector space of second order operators on $\mathbb{R}^{2}$ defined by

$$
D \in \mathcal{S}^{\operatorname{lin}} \Leftrightarrow D=h\left(-2 i \partial_{t}-\partial_{r}^{2}\right)+V(t, r), \quad h, V \in C^{\infty}\left(\mathbb{R}^{2}\right)
$$

and $\mathcal{S}^{\text {aff }} \subset \mathcal{S}^{\text {lin }}$ the affine subspace of "Schrödinger operators" given by the hyperplane $h=1$.

In other words, an element of $\mathcal{S}^{\text {aff }}$ is the sum of the free Schrödinger operator $-2 i \partial_{t}-\partial_{r}^{2}$ and of a potential $V$.

Proposition 1.5. (see [31], Propositions 2.5 and 2.6) Let $\sigma_{1 / 4}: S V \rightarrow \operatorname{Hom}\left(\mathcal{S}^{\text {lin }}\right.$, $\left.\mathcal{S}^{\text {lin }}\right)$ the representation of the group of $S V$ on the space of Schrödinger operators defined by the left-and-right action

$$
\sigma_{1 / 4}(g): D \rightarrow \pi_{5 / 4}(g) D \pi_{1 / 4}(g)^{-1}, \quad g \in S V, \quad D \in \mathcal{S}^{\text {lin }} .
$$

Then $\sigma_{1 / 4}$ restricts to an affine action on the affine subspace $\mathcal{S}^{\text {aff }}$ which is given by the following formulas:

$$
\begin{aligned}
& \sigma_{1 / 4}(\phi ; 0) \cdot\left(-2 i \partial_{t}-\partial_{r}^{2}+V(t, r)\right) \\
& \quad=-2 i \partial_{t}-\partial_{r}^{2}+\phi^{\prime}(t) V\left(\phi(t), r \sqrt{\phi^{\prime}(t)}\right)+\frac{1}{2} r^{2} \Theta(\phi)(t), \\
& \sigma_{1 / 4}(1 ;(a, b)) \cdot\left(-2 i \partial_{t}-\partial_{r}^{2}+V(t, r)\right) \\
& \quad=-2 i \partial_{t}-\partial_{r}^{2}+V(t, r-a(t))-2 r a^{\prime \prime}(t)-\left(2 b^{\prime}(t)-a(t) a^{\prime \prime}(t)\right),
\end{aligned}
$$

where $\Theta: \phi \rightarrow \frac{\phi^{\prime \prime \prime}}{\phi^{\prime}}-\frac{3}{2}\left(\frac{\phi^{\prime \prime}}{\phi^{\prime}}\right)^{2}$ is the Schwarzian derivative.

One may also consider a generalized left-and-right action $\sigma_{\lambda}(g): D \rightarrow \pi_{\lambda+1}(g) D$ $\pi_{\lambda}(g)^{-1}$, but then the subspace $\mathcal{S}_{2}^{\text {aff }}$ (see Definition 2.1.2) is not preserved by $\left.\sigma_{\lambda}\right|_{\text {Diff }_{+}(\mathbb{R} / 2 \pi \mathbb{Z})}$ anymore, which ruins all subsequent computations. Actually $1 / 4$ corresponds to the "scaling dimension" of the Schrödingerian field in one dimension (see [32]).

We shall occasionally use the time-reparametrization

$$
\phi: \mathbb{R} / 2 \pi \mathbb{Z} \rightarrow S^{1} \simeq U(1), \quad \theta \rightarrow t=e^{i \theta}
$$

from the torus to the unit circle. It allows to switch from the Fourier coordinate $\theta$ to the Laurent coordinate $t$. In particular,

$$
\begin{aligned}
\mathcal{L}_{t^{n+1}} & =\pi_{1 / 4}(\phi ; 0) \mathcal{L}_{e^{i n \theta}} \pi_{1 / 4}(\phi ; 0)^{-1} \\
\mathcal{Y}_{t^{n+\frac{1}{2}}} & =\pi_{1 / 4}(\phi ; 0) \mathcal{Y}_{e^{i n \theta}} \pi_{1 / 4}(\phi ; 0)^{-1} \\
\mathcal{M}_{t^{n}} & =\pi_{1 / 4}(\phi ; 0) \mathcal{M}_{e^{i n \theta}} \pi_{1 / 4}(\phi ; 0)^{-1}
\end{aligned}
$$

If $n$ is an integer, $\mathcal{Y}_{t^{n+\frac{1}{2}}}$ should be understood to be acting on the twofold covering of the complex plane where the square-root is defined; conversely, if $n$ is a halfinteger, then $\mathcal{Y}_{e^{i n \theta}}$ acts on $4 \pi$-periodic functions. In other words, the "natural" choice for $\mathfrak{s v}$ should be $\mathfrak{s v}\left(\frac{1}{2}\right)$, resp. $\mathfrak{s v}(0)$ in the Laurent, resp. Fourier coordinates. 
Applying formally the formulas of Proposition 1.3, one gets

$$
\left(\pi_{1 / 4}(\phi ; 0)^{-1} f\right)(\theta, x)=\left(i e^{-i \theta}\right)^{1 / 4} e^{-\frac{1}{4} i x^{2}} f\left(e^{i \theta}, \pm x e^{i\left(\frac{\theta}{2}+\frac{\pi}{4}\right)}\right)
$$

(with some ambiguity in the sign) which is an $8 \pi$-periodic function. Applying now (still formally) Proposition 1.5 yields the following result, which can be checked by direct computation.

Lemma 1.6. Let $f(t, r)$ be a solution of the Schrödinger equation

$$
\left(-2 i \partial_{t}-\partial_{r}^{2}+V(t, r)\right) f(t, r)=0 .
$$

Then

$$
\tilde{f}:(\theta, x) \rightarrow e^{-i \theta / 4} e^{-\frac{1}{4} i x^{2}} f\left(e^{i \theta}, x e^{i(\theta / 2+\pi / 4)}\right)
$$

is a solution of the transformed Schrödinger equation

$$
\left[-2 i \partial_{\theta}-\partial_{x}^{2}+\frac{1}{4} x^{2}+i e^{i \theta} V\left(e^{i \theta}, x e^{i(\theta / 2+\pi / 4)}\right)\right] \tilde{f}(\theta, x)=0 .
$$

In the following sections, we shall (except when explicitly mentioned) always work with the Lie algebra $\mathfrak{s v}(0)$ in the Fourier coordinates $\theta, x$ (i.e. the Lie algebra generated by the $\mathcal{L}_{f}, \mathcal{Y}_{g}$ and $\mathcal{M}_{h}$ with $2 \pi$-periodic functions $\left.f, g, h\right)$, and write $\mathfrak{s v}$ instead of $\mathfrak{s v}(0)$ for simplicity.

\section{Classification of the Schrödinger Operators in $\mathcal{S}_{\leq 2}^{\text {aff }}$}

From now on, we shall concentrate on the affine subspace of Schrödinger operator with potentials which are at most quadratic in the space coordinate. As mentioned in the Introduction, this subspace is invariant under the action of SV. The purpose of this section is to classify the orbits.

\subsection{Statement of the problem and connection with the classification of Hill operators}

Let us first define two natural subspaces of $\mathcal{S}^{\text {aff }}$.

Definition 2.1.1. (Schrödinger operators with at most quadratic potential) (see [31], Proposition 2.6) Let $\mathcal{S}_{\leq 2}^{\text {aff }}=\left\{-2 i \partial_{\theta}-\partial_{x}^{2}+V_{2}(\theta) x^{2}+V_{1}(\theta) x+V_{0}(\theta)\right\} \subset \mathcal{S}^{\text {aff }}$ be the affine space of Schrödinger operators with a potential which is $2 \pi$-periodic in time and at most quadratic in the coordinate $x$.

Definition 2.1.2. (Schrödinger operators with quadratic potential) Let $\mathcal{S}_{2}^{\text {aff }}=$ $\left\{-2 i \partial_{\theta}-\partial_{x}^{2}+V_{2}(\theta) x^{2}\right\} \subset \mathcal{S}_{\leq 2}^{\text {aff }}$ be the affine space of Schrödinger operators in $\mathcal{S}_{\leq 2}^{\text {aff }}$ with time-periodic potential proportional to $x^{2}$.

We do not assume $V_{2}$ to be positive. Hence what we really consider are harmonic "oscillators-repulsors", corresponding to the quantization of a classical oscillatorrepulsor with time-dependent Hamiltonian $\frac{1}{2}\left(p^{2}+V_{2}(\theta) x^{2}+V_{1}(\theta) x+V_{0}(\theta)\right)$. 
If $V_{1} \equiv 0$, then the classical equation of motion $\frac{d^{2} x}{d \theta^{2}}=-V_{2}(\theta) x-\frac{1}{2} V_{1}(\theta)$ has 0 as an attractive (resp. repulsive) fixed point depending on the sign of $V_{2}$. If $V_{2}$ is not of constant sign, things can be complicated; it is not clear a priori whether solutions are stable or unstable. We shall come back to this problem (which turns out to be more or less equivalent to the a priori harder quantum problem, at least as far as monodromy in concerned) in Sec. 3.2.

The first subspace $\mathcal{S}_{\leq 2}^{\text {aff }}$ is preserved by the action of SV (see Proposition 1.5) and is in some sense minimal (the SV-orbit of the free Schrödinger equation, or of the standard harmonic oscillator $-2 i \partial_{\theta}-\partial_{x}^{2}+a^{2} x^{2}$, contains "almost" all potentials which are at most quadratic in $x$ ). As we shall prove below, the orbits in $\mathcal{S}_{\leq 2}^{\text {aff }}$ have finite codimension.

Let us write down for the convenience of the reader the restriction of the action of $\sigma_{1 / 4}$ to $\mathcal{S}_{\leq 2}^{\text {aff }}$ : let $D=-2 i \partial_{\theta}-\partial_{x}^{2}+V_{2}(\theta) x^{2}+V_{1}(\theta) x+V_{0}(\theta)$, then

$$
\begin{aligned}
\sigma_{1 / 4}(\phi ; 0)(D)= & -2 i \partial_{\theta}-\partial_{x}^{2}+\left(\phi^{\prime 2} \cdot V_{2} \circ \phi+\frac{1}{2} \Theta(\phi)\right) x^{2} \\
& +\left(\phi^{\prime 3 / 2} \cdot V_{1} \circ \phi\right) x+\phi^{\prime} \cdot V_{0} \circ \phi,
\end{aligned}
$$

recall $\Theta(\phi)=\frac{\phi^{\prime \prime \prime}}{\phi^{\prime}}-\frac{3}{2}\left(\frac{\phi^{\prime \prime}}{\phi^{\prime}}\right)^{2}$ is the Schwarzian derivative, and

$$
\begin{aligned}
\sigma_{1 / 4}(1 ;(a, b))(D)= & -2 i \partial_{\theta}-\partial_{x}^{2}+V_{2} x^{2}+\left(V_{1}-2 a V_{2}-2 a^{\prime \prime}\right) x \\
& +\left(V_{0}-a V_{1}+a^{2} V_{2}-2 b^{\prime}+a a^{\prime \prime}\right)
\end{aligned}
$$

while the infinitesimal action is given by

$$
\begin{aligned}
d \sigma_{1 / 4}\left(\mathcal{L}_{f}\right)(D)= & -\left(\frac{1}{2} f^{\prime \prime \prime}+2 f^{\prime} V_{2}+f V_{2}^{\prime}\right) x^{2} \\
& -\left(f V_{1}^{\prime}+\frac{3}{2} f^{\prime} V_{1}\right) x-\left(f V_{0}^{\prime}+f^{\prime} V_{0}\right), \\
d \sigma_{1 / 4}\left(\mathcal{Y}_{g}+\mathcal{M}_{h}\right)(D)= & -2\left(g^{\prime \prime}+g V_{2}\right) x-\left(2 h^{\prime}+g V_{1}\right) .
\end{aligned}
$$

These four formulas are fundamental for most computations below, and we shall constantly refer to them.

Similarly, $\mathcal{S}_{2}^{\text {aff }}$ is preserved by the $\sigma_{1 / 4}$-action of $\operatorname{Diff}_{+}(\mathbb{R} / 2 \pi \mathbb{Z}$ ) (see Proposition 1.5). It turns out that the orbit theory for this space is equivalent to that of the Hill operators under the Virasoro group. Let us first give some notations and recall basic facts concerning Hill operators.

Definition 2.1.3. A Hill operator is a Sturm-Liouville operator on the onedimensional torus, i.e. a second-order operator of the form $\partial_{\theta}^{2}+u(\theta)$ where $u(\theta) \in C^{\infty}(\mathbb{R} / 2 \pi \mathbb{Z})$ is a $2 \pi$-periodic function.

The action of the group of time-reparametrizations on a Hill operator may be constructed as follows. Starting "naively" from the simple action of diffeomorphisms 
on functions,

$$
\psi \rightarrow \psi \circ \phi, \quad \phi \in \operatorname{Diff}_{+}(\mathbb{R} / 2 \pi \mathbb{Z}),
$$

one sees that $\left(\partial^{2}+u\right)(\psi)=0$ is equivalent to the transformed equation $\left(\partial^{2}+p(\theta) \partial+\right.$ $q(\theta))(\psi \circ \phi)=0$ if one sets $p=-\frac{\phi^{\prime \prime}}{\phi^{\prime}}$ and $q=\phi^{\prime 2} \cdot u \circ \phi$. Then one uses the following:

Definition 2.1.4. (Wilczinsky's semi-canonical form) (see Magnus-Winkler, [27], 3.1, or Guieu, [13], Proposition 2.1.1) If $\psi$ is a solution of the second-order equation $\left(\partial^{2}+p(\theta) \partial+q(\theta)\right) \psi=0$, then $\tilde{\psi}:=\lambda(\theta) \psi$ is a solution of the Hill equation $\left(\partial^{2}+u(\theta)\right) \tilde{\psi}=0$ provided

$$
\lambda(\theta)=\exp \left(\frac{1}{2} \int_{\theta_{0}}^{\theta} p(s) d s\right)
$$

for some $\theta_{0}$ and

$$
u=-\frac{1}{2} p^{\prime}-\frac{1}{4} p^{2}+q
$$

One obtains in this case $\lambda=\left(\phi^{\prime}\right)^{-1 / 2}$, and the transformed operator reads: $\partial^{2}+$ $\left(\phi^{\prime}\right)^{2} \cdot u \circ \phi+\frac{1}{2} \Theta(\phi)$, where $\Theta$ is the Schwarzian derivative. The presence of this last term shows that this transformation defines a projective action of Diff $+(\mathbb{R} / 2 \pi \mathbb{Z})$. Summarizing, one obtains:

Proposition 2.1.5. (see Guieu, [13] or Guieu-Roger, [14]) The transformation

$$
\partial^{2}+u \rightarrow \phi_{*}\left(\partial^{2}+u\right):=\partial^{2}+\left(\phi^{\prime}\right)^{2} \cdot u \circ \phi+\frac{1}{2} \Theta(\phi)
$$

defines an action of $\operatorname{Diff}_{+}(\mathbb{R} / 2 \pi \mathbb{Z})$ on the space of Hill operators, which is equivalent to the affine coadjoint action on $\mathfrak{v i r}_{\frac{1}{2}}^{*}$ (i.e. with central charge $c=\frac{1}{2}$ ). A solution of the transformed equation may be obtained from a solution $\psi$ of the initial equation $\left(\partial^{2}+u\right) \psi=0$ by setting $\phi_{*} \psi=\left(\phi^{\prime}\right)^{-\frac{1}{2}} \psi \circ \phi$. In other words, the solutions of the Hill equations behave as $\left(-\frac{1}{2}\right)$-densities.

The important remark is now the following:

Lemma 2.1.6. The above action of $\operatorname{Diff}_{+}(\mathbb{R} / 2 \pi \mathbb{Z})$ on the space of Hill operators is equivalent to the $\sigma_{1 / 4}$-action of $\operatorname{Diff}_{+}(\mathbb{R} / 2 \pi \mathbb{Z})$ on the space $\mathcal{S}_{2}^{\text {aff }}$.

Namely, Proposition 1.5 above (see also (2.1)) shows that

$$
\sigma_{1 / 4}(\phi)\left(-2 i \partial_{\theta}-\partial_{x}^{2}+V_{2}(\theta) x^{2}\right)=-2 i \partial_{\theta}-\partial_{x}^{2}+\tilde{V}_{2}(\theta) x^{2},
$$

where the potential $\tilde{V}_{2}$ is the image of $V_{2}$ (viewed as the potential of a Hill operator in the coordinate $\theta)$ by the diffeomorphism $\phi$, i.e. $\phi_{*}\left(\partial_{\theta}^{2}+V_{2}(\theta)\right)=\partial_{\theta}^{2}+\tilde{V}_{2}(\theta)$. Once again, this should not come as a surprise since the Hill equation is the semiclassical limit of the Schrödinger operator (see Sec. 3.2).

So we shall need to recall briefly the classification of the orbits of Hill operators under the Virasoro group. There are mainly three a priori different classifications, 
which of course turn out in the end to be equivalent: the first one is by the lifted monodromy of the solutions (see for instance Khesin and Wendt, [22]); the second one consists in looking for normal forms for the solutions, either an exponential form for non-vanishing solutions or a standard form for a dynamical system associated with the repartition of the zeros (see the article by Lazutkin and Pankratova, [24]); the third one, due to Kirillov (see [23]) proceeds in a more indirect way by looking at the isotropy groups. We shall need the first and the last classification for our purposes. They are the subject of the two upcoming subsections (see also [2] for a related review and application to the global Liouville equation).

\subsection{Classification of Hill operators by the lifted monodromy}

Let us now turn to the classification of the orbits under the Virasoro group of the space of Hill operators.

Consider a pair $\left(\psi_{1}, \psi_{2}\right)$ of linearly independent solutions of the Hill equation $\left(\partial^{2}+u\right) \psi=0$. It is a classical result (a particular case of Floquet's theory for Schrödinger equations with (space)-periodic potential) that

$$
\left(\begin{array}{l}
\psi_{1}(\theta+2 \pi) \\
\psi_{2}(\theta+2 \pi)
\end{array}\right)=M(u) \cdot\left(\begin{array}{l}
\psi_{1}(\theta) \\
\psi_{2}(\theta)
\end{array}\right)
$$

for a certain matrix $M(u) \in \mathrm{SL}(2, \mathbb{R})$ (independent of $\theta$ ), called the monodromy matrix. Starting from a different basis $\left(\begin{array}{c}\tilde{\psi}_{1} \\ \tilde{\psi}_{2}\end{array}\right)$, one obtains a conjugate matrix $\tilde{M}(u)$. The above action of the Virasoro group on the Hill equation leaves the monodromy matrix unchanged, as can be seen from the transformed solutions $\phi_{*} \psi_{1}, \phi_{*} \psi_{2}$. Hence the conjugacy class of the monodromy matrix is an invariant of the Hill operator under the action of the diffeomorphism group.

Floquet's theory, together with the orbit theory for $\operatorname{SL}(2, \mathbb{R})$, imply that $\partial^{2}+u$ is stable (meaning that all solutions are bounded) if $|\operatorname{Tr} M|<2$ or equivalently, if $M$ is elliptic, i.e. conjugate to a rotation matrix; unstable (meaning that all solutions are unbounded) if $|\operatorname{Tr} M|>2$ or equivalently, if $M$ is hyperbolic, i.e. conjugate to a Lorentz shift $\left(\begin{array}{cc}e^{\lambda} & e^{-\lambda}\end{array}\right), \lambda>0$. If $|\operatorname{Tr} M|=2$, then $M$ can be shown to be conjugate either to \pm Id or to the unipotent matrix $\pm\left(\begin{array}{cc}1 & 2 \pi \\ 0 & 1\end{array}\right)$; in the latter case, $\partial^{2}+u$ is semi-stable, with stable and unstable solutions. Two linearly independent $2 \pi$ - or $4 \pi$-periodic solutions exist when $M= \pm \mathrm{Id}$; only one in the unipotent case; and none in in the remaining cases.

An important result due to Lazutkin-Pankratova (see [24]) states that all stable Hill operators are conjugate by a suitable time-reparametrization to a Hill operator with constant potential $\partial^{2}+\alpha, \alpha>0$. They also distinguish between oscillating and non-oscillating equations (oscillating equations have solutions with infinitely many zeros, while non-oscillating equations have solutions with at most one zero), but we shall not need to go further into this. Let us just remark that (as they also show) non-oscillating operators are also conjugate to a Hill operator with constant potential $\partial^{2}+\alpha$, with $\alpha \leq 0$ this time. Hence operators of type II, resp. III of 
Kirillov's classification (see Definition 2.3.4 below) are exactly the unstable, resp. semi-stable oscillating operators.

A complete classification of the orbits under the action of $\operatorname{Diff}_{+}(\mathbb{R} / 2 \pi / Z)$ may be obtained by considering the lifted monodromy. Set $\left(\begin{array}{l}\psi_{1}(\theta) \\ \psi_{2}(\theta)\end{array}\right)=M(u)(\theta)\left(\begin{array}{l}\psi_{1}(0) \\ \psi_{2}(0)\end{array}\right)$. The path $\theta \rightarrow M(u)(\theta) \in \operatorname{SL}(2, \mathbb{R})$ may be lifted uniquely to a path $\theta \rightarrow$ $\tilde{M}(u)(\theta) \in \widetilde{\mathrm{SL}}(2, \mathbb{R})$ such that $M(u)(0)=\mathrm{Id}$, where $\widetilde{\mathrm{SL}}(2, \mathbb{R})$ is the universal covering of $\operatorname{SL}(2, \mathbb{R})$. This procedure defines a unique lifted monodromy matrix $\tilde{M}(u):=\tilde{M}(u)(2 \pi)$ modulo conjugacy.

The following arguments (see [22]) show briefly why this invariant suffices to characterize the orbit of $u$ under diffeomorphisms. Set $\left(\begin{array}{l}\psi_{1}(\theta) \\ \psi_{2}(\theta)\end{array}\right)=\sqrt{\xi(\theta)}\left(\begin{array}{c}\cos \omega(\theta) \\ \sin \omega(\theta)\end{array}\right)$. The Wronskian

$$
W:=\psi_{1} \psi_{2}^{\prime}-\psi_{1}^{\prime} \psi_{2}
$$

(a constant of motion) is equal to $\omega^{\prime}(\theta) \xi(\theta)$, hence $\omega^{\prime}=W / \xi$ is of constant sign, say $>0$ (by choosing $W>0$ ). By the action of $\operatorname{Diff}(\mathbb{R} / 2 \pi \mathbb{Z})$, one can arrange that $\omega^{\prime}$ is constant, while $\omega(0)$ and $\omega(2 \pi)$ remain related by the homographic action of $M(u)$, viz. $\cot \omega(2 \pi)=[a \cot \omega(0)+b] /[c \cot \omega(0)+d]$ if $M(u)=\left(\begin{array}{ll}a & b \\ c & d\end{array}\right) \in \operatorname{SL}(2, \mathbb{R})$. The lifting of the monodromy produces a supplementary invariant: the winding number $n:=\lfloor(\omega(2 \pi)-\omega(0)) / 2 \pi\rceil=\left\lfloor\frac{W}{2 \pi} \int_{0}^{2 \pi} \frac{d \theta}{\xi(\theta)}\right\rceil(\lfloor\cdot\rceil=$ entire part $)$, namely, the integer number of complete rotations made by the angle $\omega$.

This change of function is particularly relevant in the elliptic case. Choose a basis $\left(\begin{array}{l}\psi_{1} \\ \psi_{2}\end{array}\right)$ such that $M=\left(\begin{array}{cc}\cos \lambda & -\sin \lambda \\ \sin \lambda & \cos \lambda\end{array}\right)$. Then $\pm \lambda=\omega(2 \pi)-\omega(0)=W \int_{0}^{2 \pi} \frac{d \theta}{\xi(\theta)}[2 \pi]$.

If $M=\left(\begin{array}{cc}e^{\lambda} & e^{-\lambda}\end{array}\right)$ is hyperbolic instead, set rather

$$
\psi_{1}^{2}(\theta)=\frac{1}{2}|\xi(\theta)| e^{2 \omega(\theta)}, \quad \psi_{2}^{2}(\theta)=\frac{1}{2}|\xi(\theta)| e^{-2 \omega(\theta)}
$$

with $\xi(\theta)=2\left(\psi_{1} \psi_{2}\right)(\theta)$, so that $\pm \lambda=\omega(2 \pi)-\omega(0)[2 i \pi]$. Then one finds $\omega^{\prime}=-\frac{W}{\xi}$, hence $\omega=-W \int \frac{d \theta}{\xi(\theta)}$. The functions $\frac{1}{\xi}$ and $\omega$ are not well-defined if $\psi_{1}$ or $\psi_{2}$ has some zeros. Supposing $u$ is analytic, the functions $\psi_{1}, \psi_{2}$ may be extended analytically to some strip $\Omega=\{|\operatorname{Im} \theta|<\varepsilon\}$. Choose some contour $\Gamma \subset \Omega$ avoiding the zeros of $\psi_{1}$ and $\psi_{2}$ such that (assuming $\xi(0) \neq 0$, otherwise use a translation) $\Gamma(0)=0$ and $\Gamma(2 \pi)=2 \pi$. The idea is to keep $\Gamma$ real away from some symmetric neighborhood $U_{\varepsilon}$ of the zeros, and to complete the path with half-circles centered on the real axis of radius $\varepsilon$ around each zero, taken indifferently in the upper- or lower-half plane (compare with Sec. 3.2 below where more care is needed). Suppose $\psi_{1}\left(\theta_{0}\right)=0$ for instance, so $\psi_{1}^{\prime}\left(\theta_{0}\right)=a \neq 0$ and $\psi_{2}\left(\theta_{0}\right)=-\frac{W}{a}$. Then

$$
\begin{aligned}
-W \int_{\Gamma \cap\left[\theta_{0}-\varepsilon, \theta_{0}+\varepsilon\right]} \frac{d \theta}{\xi(\theta)} & =-W \int_{\theta_{0}-\varepsilon}^{\theta_{0}+\varepsilon} \frac{d \theta}{\theta-\theta_{0} \pm i 0} \frac{\theta-\theta_{0}}{\xi(\theta)} \\
& =-W p . v \cdot \int_{\theta_{0}-\varepsilon}^{\theta_{0}+\varepsilon} \frac{d \theta}{\xi(\theta)} \pm i \frac{\pi}{2}
\end{aligned}
$$


(depending on the position of the half-circle with respect to the real axis) since $\frac{1}{\theta-\theta_{0} \pm i 0}=p \cdot v \cdot \frac{1}{\theta-\theta_{0}} \mp i \pi \delta_{\theta_{0}}$ (see for instance [8]) and the residues of $\frac{1}{\xi(\theta)}$ at the zeros of $\xi$ are $\pm \frac{1}{2 W}$. It is clear from the above definitions that $\xi$ has only simple zeros, in even number. Hence $-W \int_{\Gamma} \frac{d \theta}{\xi(\theta)} \equiv-W p . v \cdot \int_{0}^{2 \pi} \frac{d \theta}{\xi(\theta)} \equiv \lambda[i \pi]$. By exponentiating, one obtains a monodromy matrix in $\operatorname{PSL}(2, \mathbb{R})=\operatorname{SL}(2, \mathbb{R}) /\{ \pm 1\}$.

Finally, if $M$ is unipotent, $M= \pm\left(\begin{array}{ll}1 & a \\ 0 & 1\end{array}\right)$ in some basis $\left(\begin{array}{l}\psi_{1} \\ \psi_{2}\end{array}\right)$, set $\psi_{1}(\theta)=\omega \psi_{2}(\theta)$ and $\xi=\psi_{2}^{2}$, so that $\omega(2 \pi)=\omega(0)+a$. Then $\omega^{\prime}=-\frac{W}{\xi}$, so $\omega$ is once again defined as $-W \int \frac{d \theta}{\xi(\theta)}$ if $\xi$ does not have any zero. In the contrary case, one uses a deformation of contour as in the hyperbolic case, to obtain

$$
-W \int_{\Gamma \cap\left[\theta_{0}-\varepsilon, \theta_{0}+\varepsilon\right]} \frac{d \theta}{\xi(\theta)}=-W \int_{\theta_{0}-\varepsilon}^{\theta_{0}+\varepsilon} \frac{d \theta}{\left(\theta-\theta_{0} \pm i 0\right)^{2}} \frac{\left(\theta-\theta_{0}\right)^{2}}{\psi_{2}^{2}(\theta)} .
$$

Since $\frac{1}{\left(\theta-\theta_{0} \pm i 0\right)^{2}}=p \cdot v \cdot \frac{1}{\left(\theta-\theta_{0}\right)^{2}} \pm i \pi \delta_{\theta_{0}}^{\prime}$ and $\frac{\left(\theta-\theta_{0}\right)^{2}}{\psi_{2}^{2}(\theta)}=1+O\left(\left(\theta-\theta_{0}\right)^{2}\right)$, we have $\psi_{2}^{\prime \prime}\left(\theta_{0}\right)=-V_{2}\left(\theta_{0}\right) \psi_{2}\left(\theta_{0}\right)=0$, the Dirac term does not make any contribution at all this time, hence

$$
a=\omega(2 \pi)-\omega(0)=-W \int_{\Gamma} \frac{d \theta}{\xi(\theta)},
$$

where $\Gamma:[0,2 \pi] \rightarrow \mathbb{C}$ is an arbitrary contour as defined above.

Summarizing:

Proposition 2.2.1. (see [22] for (ii)) (i) The lifted monodromy of the operator $\partial^{2}+u$ is characterized by the (correctly normalized) quantity $\int_{0}^{2 \pi} \frac{d \theta}{\xi(\theta)}$ or $\int_{\Gamma} \frac{d \theta}{\xi(\theta)}$, where $\xi \in \mathrm{Stab}_{u}$.

(ii) The orbits under the diffeomorphism group of the space of Hill operators are characterized by the conjugacy class of their lifted monodromy. More precisely, the lifted monodromy defines a bijection from the set of orbits onto the space of conjugacy classes of $(\widetilde{\mathrm{SL}}(2, \mathbb{R}) \backslash\{ \pm 1\}) /\{ \pm 1\}$ (an element $M \in \widetilde{\mathrm{SL}}(2, \mathbb{R}$ ) has to be identified with its opposite $-M)$.

\subsection{Kirillov's classification of Hill operators by isotropy subgroups}

Another classification, also useful for our purposes (and more explicit in some sense), is due to Kirillov. Introduce first

Definition 2.3.1. Let $\operatorname{Stab}_{u}, u \in C^{\infty}(\mathbb{R} / 2 \pi \mathbb{Z})$ be the isotropy subgroup (or stabilizer) of $\partial^{2}+u$ in $\operatorname{Diff}_{+}(\mathbb{R} / 2 \pi \mathbb{Z})$, namely,

$$
\operatorname{Stab}_{u}:=\left\{\phi \in \operatorname{Diff}_{+}(\mathbb{R} / 2 \pi \mathbb{Z}) \mid \phi_{*}\left(\partial^{2}+u\right)=\partial^{2}+u\right\} .
$$

Proposition 2.3.2. (definition of the first integral $I$ ) (see [13]) (1) Let $\xi \in$ $C^{\infty}(\mathbb{R} / 2 \pi \mathbb{Z})$ : then $\xi \in \operatorname{Lie}\left(\operatorname{Stab}_{u}\right)$ if and only if $\xi$ satisfies

$$
\frac{1}{2} \xi^{\prime \prime \prime}+2 u \xi^{\prime}+u^{\prime} \xi=0
$$


(2) Let $I_{u}(\xi):=\xi \xi^{\prime \prime}-\frac{1}{2} \xi^{\prime 2}+2 u \xi^{2}$. Then $I_{u}(\xi)$ is a constant of motion if $\xi \in$ $\operatorname{Lie}\left(\operatorname{Stab}_{u}\right)$.

(3) Consider $\phi \in \operatorname{Diff}_{+}(\mathbb{R} / 2 \pi \mathbb{Z})$ and the transformed potential $\tilde{u}$ such that $\phi_{*}\left(\partial^{2}+\right.$ $u)=\partial^{2}+\tilde{u}$. Then

$$
I_{\tilde{u}}\left(\phi^{\prime-1} \cdot \xi \circ \phi\right)=I_{u}(\xi)
$$

(4) Consider the Hill equation $\left(\partial^{2}+u\right) \psi(\theta)=0$. If $\left(\psi_{1}, \psi_{2}\right)$ is a basis of solutions of this equation, then $\xi:=a_{11} \psi_{1}^{2}+2 a_{12} \psi_{1} \psi_{2}+a_{22} \psi_{2}^{2}\left(a_{11}, a_{12}, a_{22} \in \mathbb{R}\right)$ satisfies the equation

$$
\frac{1}{2} \xi^{\prime \prime \prime}+2 u \xi^{\prime}+u^{\prime} \xi=0
$$

In other words, $\xi \in \mathrm{Stab}_{u}$ is in the isotropy subgroup of the Hill operator $\partial^{2}+u$.

Conversely, any solution of (2.16) can be obtained in this way.

(5) (Same notations) consider in particular $\xi=\psi_{1}^{2}+\psi_{2}^{2}$. Then $I_{u}(\xi)=W^{2}$ if $W$ is the Wronskian of $\left(\psi_{1}, \psi_{2}\right)$, namely, $W=\psi_{1} \psi_{2}^{\prime}-\psi_{1}^{\prime} \psi_{2}$ (constant of the motion).

Note (see $(3))$ that $\left(\phi^{\prime-1} \cdot \xi \circ \phi\right) \partial$ is the conjugate of the vector $\xi \partial \in \operatorname{Vect}\left(S^{1}\right)$ by the diffeomorphism $\phi$. Hence one may say that the first integral $I$ is invariant under the (adjoint-and-coadjoint) action of $\operatorname{Diff}_{+}(\mathbb{R} / 2 \pi \mathbb{Z})$.

Consider now the (adjoint) orbit of $\xi$ under $\operatorname{Diff}_{+}(\mathbb{R} / 2 \pi \mathbb{Z})$. Clearly, $\int_{0}^{2 \pi} \frac{d \theta}{\xi(\theta)}$ (if well-defined, i.e. if $\xi$ has no zero) does not depend on the choice of the point on the orbit since $\int_{0}^{2 \pi} \frac{d \theta}{\phi^{\prime-1}(\theta) \xi \circ \phi(\theta)}=\int_{0}^{2 \pi} \frac{d u}{\xi(u)}$. It is easy to see from Proposition 2.3.2(2) that $\xi$ either never vanishes (case I), or has an even number of simple zeros (case II), or has a finite number of double zeros (case III). Cases II, III correspond to a hyperbolic, resp. unipotent monodromy matrix (see discussion in Sec. 2.2). In case II, $I_{u}(\xi)=-\frac{1}{2} \xi^{\prime}\left(t_{0}\right)^{2}<0$ if $t_{0}$ is any zero. The principal value integral $p \cdot v \cdot \int_{0}^{2 \pi} \frac{d t}{\xi(t)}$ is well-defined. In case III, $I_{u}(\xi)=0$ and the regularized integral $\int_{\Gamma} \frac{d \theta}{\xi(\theta)}$ (see above) is well-defined and independent of the choice of the contour $\Gamma$. Note that Kirillov uses instead the following regularization, $\lim _{\varepsilon \rightarrow 0} \int_{[0,2 \pi] \backslash U_{\varepsilon}} \frac{d t}{\xi(t)}-\frac{C}{\varepsilon}$ (where $U_{\varepsilon}$ is a symmetric $\varepsilon$-neighborhood of the zeros) with $C$ chosen so that the limit is finite. The two regularizations are different. Both are perfectly satisfactory to define an invariant of the orbits, but computations show that the Berry phase is proportional to $\int \frac{d \theta}{\xi(\theta)}$.

Now the integral $\int_{0}^{2 \pi} \frac{d \theta}{\xi(\theta)}$ (cases I) and its variants for cases II, III are invariant under the diffeomorphism group. The discussion in Sec. 2.2 shows that they characterize the lifted monodromy of $\partial^{2}+u$. The invariant $I_{u}(\xi)$ is also needed to fix $u$ uniquely in case I (see Proposition 2.3.2(2)) since $\xi$ stabilizes all operators of the type $\partial^{2}+u+\frac{C}{\xi^{2}}(C \in \mathbb{R})$. It turns out that $\int_{0}^{2 \pi}$, or its variants, and $I_{u}(\xi)$ (in cases II and III), together with a discrete invariant $n \in \mathbb{N}$, suffice to distinguish between the different adjoint orbits of stabilizers (note that general adjoint orbits may be 
much more complicated, see [14]). One has the following:

Proposition 2.3.3. (Classification of the coadjoint invariants and of the orbits) (see Kirillov [23]) (1) Case I: $\xi$ is conjugate by a diffeomorphism $\phi$ to a (nonzero) constant $a \partial_{\theta}, a \neq 0$. Hence $\phi^{\prime-1} \cdot \xi \circ \phi \in \operatorname{Lie}\left(\operatorname{Stab}_{\partial^{2}+\alpha}\right)$ for a certain constant $\alpha$. The stabilizer Stab $_{\partial^{2}+\alpha}$ is:

(a) (non-generic case) either isomorphic to $\widetilde{\mathrm{SL}}^{(n)}(2, \mathbb{R})$ (the $n$-fold covering of $\operatorname{SL}(2, \mathbb{R}))$, with $\operatorname{Lie}\left(\operatorname{Stab}_{\partial^{2}+\alpha}\right)=\mathbb{R} \partial_{\theta} \oplus \mathbb{R} \cos n \theta \partial_{\theta} \oplus \mathbb{R} \sin n \theta \partial_{\theta}$ if $\alpha=\frac{n^{2}}{4}$ for some $n \in \mathbb{N}^{*}$; then the monodromy in $\operatorname{PSL}(2, \mathbb{R})=\operatorname{SL}(2, \mathbb{R}) /\{ \pm 1\}$ is trivial, while the lifted monodromy matrix is the central element in $\widetilde{\mathrm{SL}}(2, \mathbb{R}) /\{ \pm 1\}$ corresponding to a rotation of an angle $\pi n$;

(b) or (generic case) one-dimensional, equal to the rotation group Rot $\subset$ $\operatorname{Diff}_{+}(\mathbb{R} / 2 \pi \mathbb{Z})$ generated by the constant field $\partial$ in the remaining cases.

The invariants are given by $I_{u}(\xi)=2 \alpha a^{2}, \int_{0}^{2 \pi} \frac{d \theta}{\xi(\theta)}=\frac{2 \pi}{a}$. The monodromy can be in any conjugacy class of $\operatorname{PSL}(2, \mathbb{R})$ except \pm Id.

(2) Case II: $\xi$ is conjugate to the field $a \sin n \theta(1+\alpha \sin n \theta) \partial_{\theta}, n=1,2, \ldots, 0 \leq$ $\alpha<1$, which stabilizes $\partial^{2}+u_{n, \alpha}$, where

$$
u_{n, \alpha}(\theta):=\frac{n^{2}}{4}\left[\frac{1+6 \alpha \sin n \theta+4 \alpha^{2} \sin ^{2} n \theta}{(1+\alpha \sin n \theta)^{2}}\right] .
$$

The monodromy matrix is hyperbolic. The invariants take the values $I_{u}(\xi)=$ $-2 a^{2} n^{2}<0, p . v \cdot \int_{0}^{2 \pi} \frac{d \theta}{\xi(\theta)}=\frac{2 \pi \alpha}{a \sqrt{1-\alpha^{2}}}$.

(3) Case III: $\xi$ is conjugate to $\xi_{ \pm, n, \alpha}:= \pm(1+\sin n \theta)(1+\alpha \sin n \theta) \partial, 0 \leq \alpha<1$, corresponding to a potential $v_{n, \alpha}$,

$$
v_{n, \alpha}(\theta)=\frac{n^{2}}{4}\left[\frac{(\alpha-1)^{2}+2 \alpha(3-\alpha) \sin n \theta+4 \alpha^{2} \sin ^{2} n \theta}{(1+\alpha \sin n \theta)^{2}}\right] .
$$

The monodromy matrix is unipotent. The invariant $I_{u}(\xi)$ vanishes, while $\int_{\Gamma} \frac{d \theta}{\xi_{+}(\theta)}=$ $\frac{-2 \pi}{(1-\alpha) \sqrt{1-\alpha^{2}}}$. The discrete invariant $n$ suffices to characterize the orbit of $\partial^{2}+u$.

In cases II and III (provided $\alpha>0$ ), the stabilizer is one-dimensional, generated by $\xi \partial_{\theta}$.

In the generic cases (case $I, \alpha \neq n^{2} / 4, n=0,1, \ldots$ or case $I I$ ) the monodromy matrix is elliptic, resp. hyperbolic, if and only if $I_{u}(\xi)>0$, resp. $I_{u}(\xi)<0$. In cases $I(\alpha=0)$ and III (with unipotent monodromy), $I_{u}(\xi)=0$.

There is a mistake in Lemma 3 of [23] (the potential $u_{n, \alpha}$ given there is incorrect). The potential $v_{n, \alpha}$ was missing, together with the value of $\int_{\Gamma} \frac{d \theta}{\xi_{ \pm}(\theta)}$. Both are obtained by straightforward computations.

This classification is also natural when one thinks of the behavior of the solutions (see Lazutkin-Pankratova [24] and Sec. 2.2). In particular, case II (resp. III) correspond to operators with unstable (resp. semi-stable), oscillating solutions, while case I corresponds to operators with stable, oscillating solutions $(\alpha>0)$, 
resp. unstable, non-oscillating solutions $(\alpha<0)$, resp. semi-stable, non-oscillating solutions $(\alpha=0)$.

Note that in the case I generic, the three-dimensional isotropy subalgebra contains fields $\xi$ of types I, II $(\alpha=0)$ and III $(\alpha=0)$, hence the following nomenclature:

Definition 2.3.4. If $\partial^{2}+u$ has a stabilizer $\xi$ of types I, or of types II, III with $\alpha=0$, then $\partial^{2}+u$ may be turned into a Hill operator with constant potential, and we shall say that the operator $\partial^{2}+u$ (or the potential $u$ ) is of type I. If $\partial^{2}+u$ has a stabilizer of type II, resp. III with $\alpha \neq 0$, then we shall say that $\partial^{2}+u$ and $u$ are of type II, resp. type III.

Similarly, we shall say that the Schrödinger operator $-2 i \partial_{\theta}-\partial_{x}^{2}+V_{2}(\theta) x^{2}+$ $V_{1}(\theta) x+V_{0}(\theta)$ is of type I (resp. II, III) if the Hill operator $\partial_{\theta}^{2}+V_{2}(\theta)$ is of the corresponding type.

Note that the cases I generic $\left(\alpha \neq \frac{n^{2}}{4}, n=0,1, \ldots\right)$ and II are generic (i.e. dense in $\mathcal{S}_{\leq 2}^{\text {aff }}$ ).

Now the eigenvalues of the monodromy matrix (and also the lifted monodromy) can easily be obtained once one knows the values of the invariants $\int_{0}^{2 \pi} \frac{d \theta}{\xi(\theta)}$ and $I_{u}(\xi)$. The following lemma gives the link between the two classifications:

Lemma 2.3.5. Suppose $D=\partial^{2}+u$ is of type $I$ (with $\alpha \neq 0$ ) or II (i.e. its monodromy is either elliptic or hyperbolic). If $D$ is of type I non-generic, conjugate to $\partial^{2}+n^{2} / 4$ for some $n \geq 1$, choose $\xi$ to be conjugate to some nonzero multiple of $\partial_{\theta}$. Now (in all cases) normalize $\xi$ by requiring that $I_{u}(\xi)=2$, so that $\xi$ is real in the elliptic case and purely imaginary in the hyperbolic case. Then the eigenvalues of the monodromy matrix are given by $\exp \pm i \int_{0}^{2 \pi} \frac{d \theta}{\xi(\theta)}$ or $\exp \pm i p \cdot v \cdot \int_{0}^{2 \pi} \frac{d \theta}{\xi(\theta)}$.

Proof. Coming back to the discussion in Sec. 2.2, one checks easily (with the normalization chosen there) that $I_{u}(\xi)=2 W^{2}$ in the elliptic case, and $I_{u}(\xi)=$ $-2 W^{2}$ in the hyperbolic case. Choose a basis of solutions $\left(\psi_{1}, \psi_{2}\right)$ such that $W=1$ and multiply $\xi$ by $i$ in the hyperbolic case. Then (in both cases) the eigenvalues of the monodromy matrix ( $\pm i \lambda$ in the elliptic case, and $\pm \lambda$ in the hyperbolic case) are given by $\exp \pm i \int_{0}^{2 \pi} \frac{d \theta}{\xi(\theta)}$ or the exponential of the corresponding principal value integral.

\subsection{Classification of the $S V$-orbits in $\mathcal{S}_{\leq 2}^{\text {aff }}$}

This problem can be solved by extending the above results, which may be interpreted as the decomposition of $\mathcal{S}_{2}^{\text {aff }}$ into Diff $+(\mathbb{R} / 2 \pi \mathbb{Z})$-orbits. Let us first compute the stabilizers of some operators that will be shown later to be representatives of all the orbits. We choose to present the results in the Fourier coordinates $(\theta, x)$. The orbits of type I, resp. III split into orbits of type (i), (i)bis, resp. (iii), (iii)bis due to the presence of the linear term $V_{1}(\theta) x$ in the potential. 
The computations depend on the formulas of Proposition 1.5, see formulas (2.1)(2.4) for more convenience.

Definition 2.4.1. If $D \in \mathcal{S}_{\leq 2}^{\text {aff }}$, we denote by $G_{D}$ the stabilizer of $D$ in the Schrödinger-Virasoro group SV, i.e. $G_{D}=\left\{g \in \mathrm{SV} \mid \sigma_{1 / 4}(g) \cdot D=D\right\}$.

Recall the notation $\operatorname{Stab}_{u}, u \in C^{\infty}(\mathbb{R} / 2 \pi \mathbb{Z})$ is used for the stabilizer in $\operatorname{Diff}_{+}(\mathbb{R} / 2 \pi \mathbb{Z})$ of the corresponding Hill operator.

Note that $\mathcal{M}_{1}=M_{0}$ (whose exponential amounts to the multiplication of the wave functions $\psi$ by a constant phase) acts trivially on any operator $D$, hence $\mathcal{M}_{1} \in G_{D}$ always. The rotation group $\theta \rightarrow \theta+\theta_{0}$ generated by $d \sigma_{1 / 4}\left(\mathcal{L}_{1}\right)=$ $d \sigma_{1 / 4}\left(L_{0}\right)=-\partial_{\theta}$ will be denoted by Rot.

In the following classification, we shall call harmonic oscillators (resp. harmonic repulsors) operators with elliptic, resp. hyperbolic monodromy.

(i) Time-independent harmonic oscillators or repulsors

Set $D_{\alpha, \gamma}:=-2 i \partial_{\theta}-\partial_{x}^{2}+\alpha x^{2}+\gamma(\alpha, \gamma \in \mathbb{R})$. It is clear that $L_{-1}=\partial_{\theta}$ leaves $D_{\alpha, \gamma}$ invariant in all cases. Suppose first for simplicity that $\gamma=0$. Then $G_{D}=$ $\left(G_{0}\right)_{D} \ltimes H_{D}$ (see Proposition 1.3 for notations) is a semi-direct product, so one retrieves Kirillov's results (see Proposition 2.3.3, case I) for $\left(G_{0}\right)_{D}$; to be specific, $\operatorname{Lie}\left(\left(G_{0}\right)_{D_{n^{2} / 4,0}}\right)=\mathbb{R} \partial_{\theta} \oplus \mathbb{R} \mathcal{L}_{\sin n \theta} \oplus \mathbb{R} \mathcal{L}_{\cos n \theta}$ if $n \in \mathbb{N}^{*}$, and $\operatorname{Lie}\left(\left(G_{0}\right)_{D_{\alpha, 0}}\right)=\mathbb{R} \partial_{\theta}$ otherwise.

Now $(1 ;(a, b)) \in H_{D_{\alpha, 0}}$ if and only if $b^{\prime}=0$ and $a^{\prime \prime}=-\alpha a$. The latter equation has a nontrivial solution if and only if $\alpha=0$ (in which case $\operatorname{Lie}\left(H_{D}\right)=$ $\left.\mathbb{R} \mathcal{Y}_{1} \oplus \mathbb{R} \mathcal{M}_{1}\right)$ or $\alpha=n^{2} / 4, n \geq 1$ with $n$ even, in which case $\operatorname{Lie}\left(H_{D}\right)=$ $\mathbb{R} \mathcal{Y}_{\cos n \theta / 2} \oplus \mathbb{R} \mathcal{Y}_{\sin n \theta / 2} \oplus \mathcal{M}_{1}$. Then $\exp \frac{1}{n} \mathcal{L}_{1} \subset \widetilde{\mathrm{SL}^{(n)}}(2, \mathbb{R})$ is the rotation of angle $2 \pi$, while $\left.\exp \frac{1}{n} \operatorname{ad} \mathcal{L}_{1}\right|_{\left[\operatorname{Lie}\left(\mathcal{H}_{D}\right), \operatorname{Lie}\left(\mathcal{H}_{D}\right)\right]}$ is a rotation of angle $\pi$.

The isotropy groups $G_{D}$ are the same in the case $\gamma \neq 0$, except for a different embedding involving sometimes complicated components in the nilpotent part of SV which do not change the commutation relations (so that $G_{D}$ is no more a semi-direct product $\left.\left(G_{0}\right)_{D} \ltimes H_{D}\right)$.

Altogether, one has proved:

Theorem 2.4.2. (1) If $\alpha=n^{2} / 4$, where $n \geq 2$ is an even integer, then $G_{D_{n^{2} / 4, \gamma}} \simeq \widetilde{\mathrm{SL}}^{(n)}(2, \mathbb{R}) \ltimes \mathcal{H}_{1}$ is isomorphic to an $n$-covering of the Schrödinger group; the semi-direct action of $\widetilde{\mathrm{SL}}^{(n)}(2, \mathbb{R})$ quotients out into an action of the two-fold covering $\widetilde{\mathrm{SL}}{ }^{(2)}(2, \mathbb{R})$. The Lie algebra of the group $\widetilde{\mathrm{SL}}^{(n)}(2, \mathbb{R})$ acts as $\mathbb{R} \partial_{\theta} \oplus \mathbb{R}\left(\mathcal{L}_{\sin n \theta}+\mathcal{M}_{-\frac{1}{2} \gamma \sin n \theta}\right) \oplus \mathbb{R}\left(\mathcal{L}_{\cos n \theta}+\mathcal{M}_{-\frac{1}{2} \gamma \cos n \theta}\right)$. After transformation to the Laurent coordinates $(t, r)$ (and supposing $\gamma=0), G_{D_{n^{2} / 4,0}}$ is the connected Lie group with Lie algebra $\left\langle L_{0}, L_{ \pm n}\right\rangle \ltimes\left\langle Y_{ \pm n / 2}, M_{0}\right\rangle \subset \mathfrak{s v}(0)$.

(2) If $\alpha=n^{2} / 4$, where $n \geq 1$ is odd, then $G_{D_{n^{2} / 4,0}} \simeq \widetilde{\mathrm{SL}}^{(n)}(2, \mathbb{R}) \times \exp \mathbb{R} \mathcal{M}_{1}$.

(3) If $\alpha=0$, then $G_{D_{0, \gamma}}=\operatorname{Rot} \times \exp \left(\mathbb{R} \mathcal{Y}_{1} \oplus \mathbb{R} \mathcal{M}_{1}\right) \simeq(\mathbb{R} / 2 \pi \mathbb{Z}) \times \mathbb{R} \times(\mathbb{R} / 2 \pi \mathbb{Z})$ is the commutative group of constant translations-phases. After transformation 
to the Laurent coordinates $(t, r)$, it is the connected Lie group with Lie algebra $\left\langle L_{0}, Y_{0}, M_{0}\right\rangle \subset \mathfrak{s v}(0)$.

(4) In the generic case $\alpha \neq n^{2} / 4, n=0,1, \ldots$ one has simply $G_{D}=\operatorname{Rot} \times$ $\exp \mathbb{R} \mathcal{M}_{1} \simeq(\mathbb{R} / 2 \pi \mathbb{Z})^{2}$.

It is natural in view of these results to consider the twofold covering $\tilde{H}^{(2)}$ of $H$ obtained by considering $4 \pi$-periodic fields. Then the stabilizer in $\widetilde{S V}^{(2)}:=G_{0} \ltimes \tilde{H}^{(2)}$ of $D_{n^{2} / 4,0}(n \geq 1$ odd $)$ is isomorphic to $\widetilde{\mathrm{SL}}^{(n)}(2, \mathbb{R}) \ltimes \mathcal{H}_{1}$ as in the case of an even index $n$. This time $\operatorname{Lie}\left(\mathcal{H}_{1}\right)=\left\langle Y_{ \pm n / 2}, M_{0}\right\rangle \subset \mathfrak{s v}\left(\frac{1}{2}\right)$.

The best-known case is $\alpha=1 / 4(n=1), \gamma=0$. In the Laurent coordinates $(t, r), D_{1 / 4,0}$ writes $-2 i \partial_{t}-\partial_{r}^{2}$, namely, it is the free Schrödinger equation. Then $\operatorname{SL}(2, \mathbb{R}) \ltimes \mathcal{H}_{1}$ acts on $D_{1 / 4,0}$ in the usual way (see formulas $(0.2)$ ) in the Laurent coordinates.

(i)bis Special time-independent harmonic oscillators with added resonant oscillating drift

Consider

$$
D=-2 i \partial_{\theta}-\partial_{x}^{2}+n^{2} x^{2}+C \cos (n \theta-\sigma / 2) \cdot x+\gamma
$$

$\left(C, \sigma, \gamma \in \mathbb{R}, C \neq 0, n \geq 1\right.$ integer). Then computations show that $G_{D} \simeq$ $\mathbb{R} \times \mathbb{R} \times \mathbb{R} / 2 \pi \mathbb{Z}$ is three-dimensional, generated by

$$
\begin{aligned}
& \mathcal{L}_{1-\cos (2 n \theta-\sigma)}+\mathcal{Y}_{\frac{C}{8 n} \sin 3(n \theta-\sigma / 2)} \\
& -\mathcal{M}_{\frac{C^{2}}{32 n^{2}}\left(\frac{\cos 4 n(\theta-\sigma / 2)}{4}+\frac{\cos (2 n \theta-\sigma)}{2}+\frac{\gamma}{2} \cos (2 n \theta-\sigma)\right)}, \\
& \mathcal{Y}_{C \sin (n \theta-\sigma / 2)}+\mathcal{M}_{\frac{C^{2}}{8 n} \cos (2 n \theta-\sigma)}
\end{aligned}
$$

and $\mathcal{M}_{1}$. One checks (by direct computation) that the value of the associated invariant $I_{n^{2}}(1-\cos (2 n \theta-\sigma))$ is 0 .

(ii) Time-dependent Ince harmonic repulsors of type II Consider

$$
D_{n, \alpha, \gamma}=-2 i \partial_{\theta}-\partial_{x}^{2}+u_{n, \alpha}(\theta) x^{2}+\gamma, \quad n=1,2, \ldots, \alpha \in(0,1)
$$

where

$$
u_{n, \alpha}(\theta)=\frac{n^{2}}{4}\left[\frac{1+6 \alpha \sin n \theta+4 \alpha^{2} \sin ^{2} n \theta}{(1+\alpha \sin n \theta)^{2}}\right] .
$$

Then (see preceding subsection) $\mathcal{L}_{\xi}-\frac{\gamma}{2} \mathcal{M}_{\xi} \in \operatorname{Lie}\left(G_{D}\right)(\xi \neq 0)$ if and only if $\xi$ is proportional to $\xi_{n, \alpha}$, with $\xi_{n, \alpha}=\sin n \theta(1+\alpha \sin n \theta) \partial_{\theta}$. Now

$$
d \sigma_{1 / 4}\left(\mathcal{Y}_{f_{1}}+\mathcal{M}_{f_{2}}\right) \cdot D=0
$$

if and only if $f_{2}^{\prime}=0$ and $f_{1}^{\prime \prime}+u_{n, \alpha} f_{1}=0$. The latter equation is known under the name of Ince's equation (see Magnus and Winkler, [27]). 
The change of variable and function $\theta \rightarrow \delta(\theta)=\frac{\pi}{4}-n \frac{\theta}{2}, f_{1}(\theta) \rightarrow y(\delta)=$ $(1+\alpha \cos 2 \delta)^{b / 4 \alpha} f_{1}(\delta(\theta))$ with $b=-2 \alpha\left[1+\frac{i \alpha}{\sqrt{1-\alpha^{2}}}\right]$ turns the above equation into the standard form

$$
(1+a \cos 2 \delta) y^{\prime \prime}+b \sin 2 \delta y^{\prime}+(c+d \cos 2 \delta) y=0
$$

with $a=\alpha, c=1-\frac{\alpha^{2}}{1-\alpha^{2}}, d=\alpha\left[3+\frac{\alpha^{2}}{1-\alpha^{2}} \mp \frac{2 i \alpha}{\sqrt{1-\alpha^{2}}}\right]$. Conditions for the coexistence of two independent periodic solutions of Ince's equation have been studied in detail. In our case, there is no periodic solution since $\partial^{2}+u_{n, \alpha}$ is unstable (see discussion in Sec. 2.2). Hence

$$
G_{D}=\exp \left(\mathbb{R}\left(\mathcal{L}_{\xi_{n, \alpha}}-\frac{\gamma}{2} \mathcal{M}_{\xi_{n, \alpha}}\right) \oplus \mathbb{R} \mathcal{M}_{1}\right) \simeq \mathbb{R} \times(\mathbb{R} / 2 \pi \mathbb{Z}) .
$$

(iii) Non-resonant time-dependent Schrödinger operators of type III

Consider

$$
D_{n, \alpha, \gamma}=2 i \partial_{\theta}-\partial_{x}^{2}+v_{n, \alpha}(\theta) x^{2}+\gamma
$$

$(n=1,2, \ldots, \alpha \in(0,1))$. See Eq. (2.18). Similarly to case (ii), $\mathcal{L}_{\xi}-\frac{\gamma}{2} \mathcal{M}_{\xi} \in$ $\operatorname{Lie}\left(G_{D}\right)(\xi \neq 0)$ if and only if $\xi$ is proportional to $\xi_{ \pm, n, \alpha}$, where $\xi_{ \pm, n, \alpha}=$ $\pm(1+\sin n \theta)(1+\alpha \sin n \theta) \partial_{\theta}$. Then $d \sigma_{1 / 4}\left(\mathcal{Y}_{f_{1}}+\mathcal{M}_{f_{2}}\right) \cdot D=0$ if and only if $f_{2}^{\prime}=0$ and $f_{1}^{\prime \prime}+v_{n, \alpha} f_{1}=0$. This is once again Ince's equation, with parameters $a=\alpha, b=-2 \alpha, c=1-2 \alpha, d=3 \alpha$. One verifies immediately that $y(\delta)=\cos \delta$ is the unique (up to a constant) periodic solution of this semi-stable Hill equation, corresponding to $f_{1}(\theta):=(1+\alpha \sin n \theta)^{1 / 2} \cos \left(\frac{\pi}{4}-\right.$ $\left.n \frac{\theta}{2}\right)$. Note that $\xi_{+, n, \alpha}=f_{1}^{2}$ (so that $f_{1}$ is, up to a sign, the unique $C^{\infty}$ square-root of $\left.\xi_{+, n, \alpha}\right)$. Hence

$$
\begin{aligned}
G_{D} & =\exp \left(\mathbb{R}\left(\mathcal{L}_{\xi_{ \pm, n, \alpha}}-\frac{\gamma}{2} \mathcal{M}_{\xi_{ \pm, n, \alpha}}\right) \oplus \mathbb{R} \mathcal{Y}_{f_{1}} \oplus \mathbb{R} \mathcal{M}_{1}\right) \\
& \simeq \mathbb{R} \times \mathbb{R} \times(\mathbb{R} / 2 \pi \mathbb{Z}) .
\end{aligned}
$$

(iii)bis Schrödinger operators of type III with added resonant drift

Consider

$$
D=-2 i \partial_{\theta}-\partial_{x}^{2}+v_{n, \alpha}(\theta) x^{2}+C(1+\alpha \sin n \theta)^{1 / 2} \cos \left(\frac{\pi}{4}-n \frac{\theta}{2}\right) x+\gamma
$$

$(C \neq 0)$ with $v_{n, \alpha}$ as in case (iii). Set $\xi(\theta)=(1+\sin n \theta)(1+\alpha \sin n \theta)$ and $f(\theta)=(1+\alpha \sin n \theta)^{1 / 2} \cos (\pi / 4-n \theta / 2)$. Recall $\xi=f^{2}$.

Suppose $\mathcal{L}_{\xi}+\mathcal{Y}_{f_{1}}+\mathcal{M}_{f_{2}}$ stabilizes $D$. Then (see (2.3))

$$
2\left(f_{1}^{\prime \prime}+v_{n, \alpha} f_{1}\right)=C\left(\xi f^{\prime}+\frac{3}{2} \xi^{\prime} f\right)=4 C f^{2} f^{\prime}
$$

and

$$
f_{2}^{\prime}=-\frac{1}{2}\left(\gamma \xi^{\prime}+C f_{1} f\right) .
$$


The kernel of the operator $\partial^{2}+v_{n, \alpha}$ is one-dimensional, generated by $f$. Hence the above Eq. (2.25) has a solution if and only if $\int_{0}^{2 \pi}\left(\xi f^{\prime}+\frac{3}{2} \xi^{\prime} f\right) f d \theta=$ 0 , which is true since $\left(\xi f^{\prime}+\frac{3}{2} \xi^{\prime} f\right) f=\left(f^{4}\right)^{\prime}$. Now Eq. (2.26) has a solution if and only if $f_{1}$ is chosen to be the unique solution orthogonal to the kernel of $\partial^{2}+v_{n, \alpha}$, namely, if $\int_{0}^{2 \pi} f_{1} f d \theta=0$.

Now

$$
d \sigma_{1 / 4}\left(\mathcal{Y}_{g_{1}}+\mathcal{M}_{g_{2}}\right) \cdot D=-2\left(g_{1}^{\prime \prime}+v_{n, \alpha} g_{1}\right) x-f g_{1}-2 g_{2}^{\prime}
$$

vanishes if and only if $g_{1}=f$ (up to a multiplicative constant) and $\int_{0}^{2 \pi} g_{1} f d \theta=0$. The two conditions are clearly incompatible.

Altogether one has proved that $G_{D}=\exp \left(\mathbb{R}\left(\mathcal{L}_{(1+\sin n \theta)(1+\alpha \sin n \theta)}+\mathcal{Y}_{f_{1}}+\mathcal{M}_{f_{2}}\right) \oplus\right.$ $\left.\mathbb{R} \mathcal{M}_{1}\right) \simeq \mathbb{R} \times \mathbb{R} / 2 \pi \mathbb{Z}$ (with $f_{1}, f_{2}$ solving Eqs. (2.25) and (2.26)) is commutative two-dimensional.

Explicit but cumbersome formulas for $f_{1}, f_{2}$ are easy to derive from the proof of Lemma 4.10 below. We shall not need them.

There remains to prove that we have classified all the orbits in $\mathcal{S}_{\leq 2}^{\text {aff }}$.

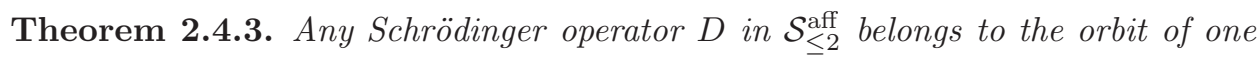
of the above operators.

Proof. Let $D \in \mathcal{S}_{\leq 2}^{\text {aff }}$. Suppose first that $V_{2}$ is of type I. Then one may assume (by a time-reparametrization) that $V_{2}=\alpha$ is a constant. The operator $D$ belongs to the orbit of $D_{\alpha, \gamma}$ (case (i)) for some $\gamma$ if and only if $V_{1}=2\left(a^{\prime \prime}+\alpha a\right)$. If $\alpha \neq n^{2}$ (or $n^{2} / 4$ if one considers the $\widetilde{S V}^{(2)}$-orbits) then this equation has a unique solution for every $V_{1}$. If $\alpha=n^{2}$, then a Fourier series $V_{1}=\sum_{k} c_{k} e^{i k \theta}$ is in the image of $\partial_{\theta}^{2}+\alpha$ if and only if $c_{ \pm n}=0$. This analysis accounts for the two cases (i) and (i)bis.

Suppose now $V_{2}$ is of type II. By a time-reparametrization one may choose $V_{2}=u_{n, \alpha}$. The operator $D$ belongs to the orbit of $D_{n, \alpha, \gamma}$ (see case (ii)) for some $\gamma$, provided $V_{1}=2\left(a^{\prime \prime}+u_{n, \alpha} a\right)$. Since $\partial^{2}+u_{n, \alpha}$ (acting on $\left.C^{\infty}(\mathbb{R} / 2 \pi \mathbb{Z})\right)$ has a trivial kernel, it has a bounded inverse and the unique solution of the above equation is $C^{\infty}$. Hence $D$ belongs to the orbit of $D_{n, \alpha, \gamma}$.

Finally, suppose $V_{2}$ is of type III. One is led to solve the equation $V_{1}=$ $2\left(a^{\prime \prime}+v_{n, \alpha} a\right)$. Recall $V_{1}(\theta)=(1+\alpha \sin n \theta)^{1 / 2} \cos \left(\frac{\pi}{4}-n \frac{\theta}{2}\right)$ solves the equation $f_{1}^{\prime \prime}+v_{n, \alpha} f_{1}=0$. Hence $V_{1}=2\left(a^{\prime \prime}+v_{n, \alpha} a\right)$ has a solution if and only if $\int_{0}^{2 \pi} V_{1}(\theta)(1+\alpha \sin n \theta)^{1 / 2} \cos \left(\frac{\pi}{4}-n \frac{\theta}{2}\right) d \theta=0$, which accounts for cases (iii) and (iii)bis.

Note that Schrödinger operators of type III are generically of type (iii)bis, and Schrödinger operators of type I with $\alpha=n^{2}, n=1,2, \ldots$ are generically of type (i)bis.

Corollary 2.4.4. For generic orbits (type (i) with $\alpha \neq \frac{n^{2}}{4}, n \geq 0$, or type (ii)), the isotropy group is two-dimensional, given by $\exp \mathbb{R}\left(\mathcal{L}_{\xi}+\mathcal{Y}_{\delta_{1}}+\mathcal{M}_{\delta_{2}}\right) \oplus \mathbb{R} \mathcal{M}_{1} \simeq$ $\mathbb{R} \times \mathbb{R} / 2 \pi \mathbb{Z}$ or $\mathbb{R} / 2 \pi \mathbb{Z} \times \mathbb{R} / 2 \pi \mathbb{Z}$ for some triple $\left(\xi, \delta_{1}, \delta_{2}\right)$ with $\xi \neq 0$. 
Let us finish with a remark. Consider a potential $V_{2}(\theta) x^{2}+V_{1}(\theta) x+V_{0}(\theta)$ of type (i), (ii) or (iii). As we shall see in the next section, the monodromy of the corresponding Schrödinger operator depends only on the (conjugacy class of the) invariant $\xi$ and the value of the constant $\gamma$ (which acts as a simple energy shift). Computing the invariant $\xi$ is a difficult task in general, but suppose it can be achieved. How does one determine the constant $\gamma$ ? We give an answer for generic elliptic or hyperbolic potentials of type (i).

Lemma 2.4.5. Let $D=-2 i \partial_{\theta}-\partial_{x}^{2}+V_{2}(\theta) x^{2}+V_{1}(\theta) x+V_{0}(\theta)$ be of type (i), elliptic or hyperbolic, generic, so that $D$ is conjugate to a unique operator $D_{\alpha, \gamma}=$ $-2 i \partial_{\theta}-\partial_{x}^{2}+\alpha x^{2}+\gamma\left(\alpha \in \mathbb{R}, \alpha \neq \frac{n^{2}}{4}, n=0,1, \ldots\right)$. Then $\gamma$ may be retrieved from

$$
\gamma=\frac{1}{2 \pi} \int_{0}^{2 \pi}\left(V_{0}-\frac{1}{4} V_{1} W_{1}\right)(\theta) d \theta,
$$

where $W_{1}$ is the unique solution of the equation $\left(\partial^{2}+V_{2}\right) W_{1}=V_{1}$.

Proof. Start from the model operator $D_{\alpha, \gamma}$, with stabilizer $\xi=1$, and apply successively $\sigma_{1 / 4}(\phi ;(0,0))$ and $\sigma_{1 / 4}(1 ;(g, h))$. Then one obtains the operator

$$
D:=-2 i \partial_{\theta}-\partial_{x}^{2}+V_{2}(\theta) x^{2}-2\left(\left(\partial^{2}+V_{2}\right) g\right) x+\left(\gamma \dot{\phi}+g\left(\partial^{2}+V_{2}\right) g-2 \dot{h}\right)
$$

(see formulas $(2.1)$ and $(2.2)$ ). Now $\int_{0}^{2 \pi} \dot{\phi}(\theta) d \theta=2 \pi$ since $\phi \in \operatorname{Diff}_{+}(\mathbb{R} / 2 \pi \mathbb{Z})$. Hence the result.

\subsection{Connection to Niederer's results}

We are referring to a classical paper by Niederer (see [28]) concerning the maximal groups of Lie symmetries of Schrödinger equations with arbitrary potentials. One may rephrase his main result as follows (though the Schrödinger-Virasoro had not been introduced at that time). Niederer shows that any transformation

$$
\psi(t, r) \rightarrow \tilde{\psi}(t, r)=\exp i f_{g}\left(g^{-1}(t, r)\right) \psi\left(g^{-1}(t, r)\right),
$$

(where $g:(t, r) \rightarrow\left(t^{\prime}, r^{\prime}\right)$ is an arbitrary coordinate transformation and $f_{g}$ an arbitrary "companion function" corresponding to a projective action) carrying the space of solutions of the Schrödinger equation

$$
\left(-2 i \partial_{t}-\partial_{r}^{2}+V(t, r)\right) \psi(t, r)=0
$$

into itself in necessarily of the form $\sigma_{1 / 4}(g)$ for some $g \in \mathrm{SV}$. This is, by the way, an elegant way of introducing the SV group in the first place. Then Niederer gives a necessary and sufficient condition for $g \in \sigma_{1 / 4}(S V)$ to leave (2.29) invariant, and produces some physically interesting examples. Let us analyze some of these examples from our point of view. It should be understood that Niederer's examples 
are given in the Laurent coordinates $(t, r)$ and hence should be transformed by using Lemma 1.6 to compare with our results.

(i) $V=0$ (free Schrödinger equation): this case corresponds, after the transformation at the end of Sec. 1 to the potential $V(\theta, x)=\frac{1}{4} x^{2}$, with invariance under the full Schrödinger group (see case (i) in Sec. 2.4, with $\alpha=1 / 4$ and $\gamma=0)$.

(ii) $V=-g r$ (free fall) corresponds to $V(\theta, x)=\frac{1}{4} x^{2}-g e^{i(\theta / 2+3 \pi / 4)} x$ (a $4 \pi$-periodic potential), which belongs to the same orbit as case (i) (free Schrödinger equation in the Laurent coordinates).

(iii) $V=\frac{1}{2} \omega^{2} r^{2}$ (harmonic oscillator) may be obtained from the free Schrödinger equation by the time reparametrization $t(u)=\tan \omega u$ for which the Schwarzian derivative is a constant, $\Theta(t)=2 \omega^{2}$ (see formulas in Proposition 1.5).

(iv) $V=k / r^{2}$ (inverse-square potential), corresponding to the operator $-2 i \partial_{\theta}-$ $\partial_{x}^{2}+\frac{x^{2}}{4}+k x^{-2}$ (harmonic oscillator with added inverse-square potential) in the Fourier coordinate. The operator is not in $\mathcal{S}_{\leq 2}^{\text {aff }}$, but the (time-independent) inverse-square potential is interesting in that this is the only potential left invariant by all transformations $V(t, r) \rightarrow \phi^{\prime}(t) V\left(\phi(t), r \sqrt{\phi^{\prime}(t)}\right.$ ) (see formulas in Proposition 1.5). So this equation is invariant by the kernel of the Schwarzian derivative, i.e. by the homographic transformations.

\section{Monodromy of Time-Dependent Schrödinger Operators of Non-Resonant Types and Ermakov-Lewis Invariants}

We "solve" in this section all Schrödinger operators in $\mathcal{S}_{\leq 2}^{\text {aff }}$ of class (i), (ii) or (iii) by using the Ermakov-Lewis invariants, to be introduced below. Since any such operator is conjugate to an operator of the type $-2 i \partial_{\theta}-\partial_{x}^{2}+V_{2}(\theta) x^{2}+\gamma(\gamma$ constant), and $\gamma$ corresponds to a simple energy shift, we shall implicitly assume that the potential is simply quadratic $\left(V_{1}=V_{0}=0\right)$.

Lemma 3.1.2 and Proposition 3.1.4 yield explicitly an evolution operator $U\left(\theta, \theta_{0}\right)$, i.e. a unitary operator on $L^{2}(\mathbb{R})$ which gives the evolution of the solutions of the Schrödinger equation from time $\theta_{0}$ to time $\theta$. This operator gives the unique solution to the Cauchy problem and allows to compute the (exact) Berry phase. The arguments in Lemmas 3.1.1, 3.1.2 and Proposition 3.1.4 are reproduced from the paper of Lewis and Riesenfeld ([26]). Unfortunately this method gives the monodromy only in the elliptic case (i.e. for operators of class (i) with $\alpha>0$ ). So we generalize their invariants to the hyperbolic and unipotent case; the invariant we must choose in order to be able to compute the monodromy is not a harmonic oscillator any more, but an operator with absolutely continuous spectrum. Nevertheless, it turns out that there does exist a phase operator, given in terms of the (possibly regularized) integral $\int_{0}^{2 \pi} \frac{d \theta}{\xi(\theta)}$ for a certain stabilizer $\xi$ of the quadratic part of the potential. The key point in order to get the whole picture is to make the bridge between Kirillov's results and the Ermakov-Lewis invariants. 


\subsection{Ermakov-Lewis invariants and Schrödinger-Virasoro invariance}

Let $H=\frac{1}{2}\left(-\partial_{x}^{2}+V_{2}(\theta) x^{2}\right)$ be the (quantum) Hamiltonian corresponding to a timedependent harmonic oscillator. The evolution of the wave function $\psi(\theta, x)$ is given by: $i \partial_{\theta} \psi(\theta, x)=H \psi(\theta, x)$, or $D \psi=0$ where $D=-2 i \partial_{\theta}+2 H=-2 i \partial_{\theta}-\partial_{x}^{2}+$ $V_{2}(\theta) x^{2}$.

The Ermakov-Lewis dynamical invariants were invented in order to find the solutions of the above equation. The idea is simple. Suppose $I(\theta, x)$ is a timedependent hermitian operator of the form $\sum_{j=0}^{N} I_{j}(\theta, x) \partial_{x}^{j}$ which is an invariant of the motion, i.e. $\frac{d}{d t} I=\partial_{t} I+\frac{1}{i}[I, H]=0$. Suppose also that, for every fixed value of $\theta, I(\theta, x)$ (defined on an appropriately defined dense subspace of $L^{2}(\mathbb{R}, d x)$, for instance on the space of test functions) is essentially self-adjoint and has a purely point spectrum. For simplicity, we shall assume that all multiplicities are one, and that one may choose normalized eigenvectors which depend regularly on $\theta$, namely,

$$
I(\theta, x) h_{n}(\theta, x)=\lambda_{n}(\theta) h_{n}(\theta, x)
$$

and $\int_{\mathbb{R}}\left|h_{n}(\theta, x)\right|^{2} d x=1$. The fact that $I$ is an invariant of the motion implies by definition that $I \psi$ is a solution of the Schrödinger equation if $\psi$ is. The following lemma shows how to solve the Schrödinger equation by means of the invariant $I$ :

Lemma 3.1.1. (see [26]) (1) The eigenvalues $\lambda_{n}(\theta)$ are constants, i.e. they do not depend on time.

(2) If $n \neq m$, then $\left\langle h_{m}(\theta),\left(i \partial_{\theta}-H\right) h_{n}(\theta)\right\rangle=0$.

Proof. (i) Applying the invariance property $\frac{\partial I}{\partial \theta}+\frac{1}{i}[I(\theta), H(\theta)]=0$ to the eigenvector $h_{n}(\theta)$ yields

$$
\frac{\partial I}{\partial \theta} h_{n}(\theta)+\frac{1}{i}\left(I(\theta)-\lambda_{n}(\theta)\right) H(\theta) h_{n}(\theta)=0 .
$$

Taking the scalar product with $h_{m}(\theta)$ gives a first equation,

$$
\left\langle h_{m}(\theta), \frac{\partial I}{\partial \theta} h_{n}(\theta)\right\rangle+\frac{1}{i}\left(\lambda_{m}(\theta)-\lambda_{n}(\theta)\right)\left\langle h_{m}(\theta), H(\theta) h_{n}(\theta)\right\rangle=0 .
$$

The eigenvalue equation $I(\theta) h_{n}(\theta)=\lambda_{n}(\theta) h_{n}(\theta)$ gives after time differentiation a second equation, namely

$$
\frac{\partial I}{\partial \theta} h_{n}(\theta)+\left(I(\theta)-\lambda_{n}(\theta)\right) \dot{h}_{n}(\theta)=\dot{\lambda}_{n}(\theta) h_{n}(\theta) .
$$

Combining Eqs. (3.2) and (3.3) for $n=m$ yields $\dot{\lambda}_{n}(\theta)=0$.

(ii) Combining this time Eqs. (3.2) and (3.3) for $n \neq m$ yields the desired equality. 
The above lemma shows that one may choose eigenvectors $h_{n}(\theta)$ that satisfy the Schrödinger equation by multiplying them by an appropriate time-dependent phase, which is the content of the following lemma.

Lemma 3.1.2. Let, for each $n, \alpha_{n}(\theta)$ be a solution of the equation

$$
\frac{d \alpha_{n}}{d \theta}=\left\langle h_{n}(\theta),\left(i \partial_{\theta}-H\right) h_{n}(\theta)\right\rangle
$$

Then the gauge-transformed eigenvectors for the invariant I

$$
\tilde{h}_{n}(\theta)=e^{i \alpha_{n}(\theta)} h_{n}(\theta)
$$

are solutions of the Schrödinger equation.

In other words, the general solution of the Schrödinger equation is:

$$
\psi(\theta):=\sum_{n} c_{n} e^{i \alpha_{n}(\theta)} h_{n}(\theta)
$$

where $c_{n}$ are constant (time-independent) coefficients.

Let us specialize to the case when $H$ is a time-dependent harmonic oscillator as above, i.e. $H=\frac{1}{2}\left(-\partial_{x}^{2}+V_{2}(\theta) x^{2}\right)$. A natural idea is to assume the following Ansatz

$$
I(\theta)=\frac{1}{2}\left[-b(\theta) \partial_{x}^{2}+a(\theta) x^{2}-i c(\theta)\left(x \partial_{x}+\partial_{x} x\right)\right] .
$$

This problem has a unique family of nontrivial solutions:

Definition 3.1.3. (Pinney-Milne equation) The nonlinear equation

$$
\ddot{\zeta}+f(\theta) \zeta-\frac{K}{\zeta^{3}}=0
$$

$(K>0)$ is called a Pinney-Milne equation. If $K=1$, then we shall say that (3.7) is a normalized Pinney-Milne equation.

Of course, every Pinney-Milne equation can easily be normalized by multiplying the function by the constant factor $K^{1 / 4}$.

The following proposition summarizes results due to Lewis and Riesenfeld (see $[26])$.

Proposition 3.1.4. (Ermakov-Lewis invariants for time-dependent harmonic oscillators) (1) The second-order operator $\mathcal{E} \mathcal{L}\left(\zeta^{2}\right)$

$$
\mathcal{E} \mathcal{L}\left(\zeta^{2}\right)(\theta)=\frac{1}{2}\left[\frac{x^{2}}{\zeta^{2}}+\left(i \zeta(\theta) \partial_{x}+\dot{\zeta}(\theta) x\right)^{2}\right]
$$

is an invariant of the time-dependent harmonic oscillator $-2 i \partial_{\theta}-\partial_{x}^{2}+V_{2}(\theta) x^{2}$ provided $\zeta$ is a solution of the following normalized Pinney-Milne equation:

$$
\ddot{\zeta}+V_{2}(\theta) \zeta-\frac{1}{\zeta^{3}}=0 .
$$


Setting $\xi=\zeta^{2}$, one may also write equivalently

$$
\begin{aligned}
\mathcal{E} \mathcal{L}(\xi)(\theta) & =\frac{1}{2 \xi}\left[x^{2}+\left(i \xi \partial_{x}+\frac{1}{2} \dot{\xi} x\right)^{2}\right] \\
& =\frac{1}{2 \xi}\left[-\xi^{2} \partial_{x}^{2}+\left(1+\frac{\dot{\xi}^{2}}{4}\right) x^{2}+\frac{i}{2} \xi \dot{\xi}\left(x \partial_{x}+\partial_{x} x\right)\right] .
\end{aligned}
$$

(2) Set

$$
a(\theta):=\frac{1}{\sqrt{2}}\left[\frac{x}{\zeta(\theta)}-\left(\zeta(\theta) \partial_{x}+i \dot{\zeta}(\theta) x\right)\right]
$$

and

$$
a^{*}(\theta)=\frac{1}{\sqrt{2}}\left[\frac{x}{\zeta(\theta)}+\left(\zeta(\theta) \partial_{x}+i \dot{\zeta}(\theta) x\right)\right]
$$

(formal adjoint of the operator $a(\theta)$ ). Then

$$
\mathcal{E} \mathcal{L}(\xi)(\theta)=a^{*}(\theta) a(\theta)+\frac{1}{2} .
$$

In other words, for every fixed value of $\theta$, the operators $a(\theta), a^{*}(\theta)$ play the roles of an annihilation, resp. creation operator for the (time-dependent) harmonic oscillator $\mathcal{E} \mathcal{L}(\xi)$.

(3) The normalized ground state of the operator $a(\theta)$ is

$$
h_{0}(\theta)=\frac{1}{2 \sqrt{\pi}} \frac{1}{\sqrt{\xi(\theta)}} \exp \left(\left(-\frac{1}{2} \frac{1}{\xi(\theta)}+\frac{i}{2}(\dot{\xi} / \xi)(\theta)\right) x^{2}\right) .
$$

(4) The solutions of Eq. (3.4) giving the phase evolution of the solutions of the Schrödinger equation are given by

$$
\alpha_{n}(\theta)=-\left(n+\frac{1}{2}\right) \int^{\theta} \frac{d \theta^{\prime}}{\xi\left(\theta^{\prime}\right)}
$$

provided one chooses the time-evolution of the eigenstates $h_{n}$ by setting

$$
\left\langle h_{n}, \partial_{\theta} h_{n}\right\rangle=\frac{i}{2}\left(n+\frac{1}{2}\right)\left(\zeta \ddot{\zeta}-\dot{\zeta}^{2}\right) .
$$

The above choice for the time-evolution of the eigenstates appears natural if one requires the standard lowering and raising relations $a(\theta) h_{n}(\theta)=n^{1 / 2} h_{n-1}(\theta)$, $a^{*}(\theta) h_{n}(\theta)=(n+1)^{1 / 2} h_{n+1}(\theta)$. Then computations show that

$$
\left\langle h_{n}, \partial_{\theta} h_{n}\right\rangle=\left\langle h_{0}, \partial_{\theta} h_{0}\right\rangle+i \frac{n}{2}\left(\zeta \ddot{\zeta}-\dot{\zeta}^{2}\right) .
$$

Hence there only remains to choose the time-evolution of the ground-state $h_{0}$. This particular choice leads to the $\left(n+\frac{1}{2}\right)$-factor typical of the spectrum of the harmonic oscillator. Note that the $h_{n}(\theta)$ do not satisfy the gauge-fixing condition typical of the adiabatic approximation (see Joye [21] for instance). But this phase choice leads to a nice interpretation of the phases $\alpha_{n}$ (up to a constant) as a canonical 
coordinate conjugate to the classical invariant $\mathcal{E} \mathcal{L}_{c l}$ (see Lemma 3.2.2 below) for the corresponding classical problem, in the generalized symplectic formalism for which time is a coordinate, so that the problem becomes autonomous (see LewisRiesenfeld [26]; see also Sec. 4 for the symplectic formalism). Also, as mentioned in the Introduction, the natural time-scale (both for the classical and the quantum problem) is $\tau(\theta):=\int^{\theta} \frac{d u}{\xi(u)}$.

The connection with the preceding sections is given by the following classical lemma (see [27], Chap. 3), which is an easy corollary of Proposition 2.3.2:

Lemma 3.1.5. (1) Let $\xi$ be a (non-necessarily periodic) solution of the equation

$$
\frac{1}{2} \xi^{\prime \prime \prime}+2 u \xi^{\prime}+u^{\prime} \xi=0
$$

so that $\xi$ stabilizes $\partial^{2}+u$. Then $\zeta:=\sqrt{\xi}$ is a solution of the Pinney-Milne equation

$$
\zeta^{\prime \prime}+u(\theta) \zeta-\frac{I_{u}(\xi) / 2}{\zeta^{3}}=0,
$$

where $I_{u}(\xi):=\xi \xi^{\prime \prime}-\frac{1}{2} \xi^{\prime 2}+2 u \xi^{2}$ is the constant defined in Proposition 2.3.2(2).

In particular, if $\xi=\psi_{1}^{2}+\psi_{2}^{2}$, where $\left(\psi_{1}, \psi_{2}\right)$ is a basis of solutions of the Hill equation $\left(\partial^{2}+u\right) \psi=0$ and $\zeta=\sqrt{\xi}$, then

$$
\zeta^{\prime \prime}+u(\theta) \zeta-\frac{W^{2}}{\zeta^{3}}=0,
$$

where $W:=\psi_{1} \psi_{2}^{\prime}-\psi_{1}^{\prime} \psi_{2}$ is the Wronskian of the two solutions.

(2) Consider $\xi \in \mathrm{Stab}_{u}$ such that $\zeta=\sqrt{\xi}$ satisfies the Pinney-Milne equation (3.18), and a time-reparametrization $\phi$. Then $\tilde{\xi}:=\phi^{\prime-1} \cdot \xi \circ \phi$ is a stabilizer of $\partial^{2}+\tilde{u}:=\phi_{*}\left(\partial^{2}+u\right)$ and $\tilde{\zeta}:=\sqrt{\tilde{\xi}}$ satisfies the transformed Pinney-Milne equation

$$
\tilde{\zeta}^{\prime \prime}+\tilde{u} \tilde{\zeta}-\frac{W^{2}}{\tilde{\zeta}^{3}}=0
$$

for the same constant $W$.

The interesting point now is that one can choose the Ermakov-Lewis invariant in such a way that the invariant associated to the image of the time-dependent harmonic oscillator $D$ by a time reparametrization (through the representation $\sigma_{1 / 4}$ ) is its image by a very natural transformation (essentially, by the corresponding change of coordinates). This provides an elegant, natural explanation for the complicated-looking phase appearing in the formulas for $\sigma_{1 / 4}$.

Theorem 3.1.6. Let $D:=-2 i \partial_{\theta}-\partial_{x}^{2}+V_{2}(\theta) x^{2}$ be a time-dependent harmonic oscillator, $\zeta$ satisfy the Pinney equation $\zeta^{\prime \prime}+V_{2} \zeta-\frac{1}{\zeta^{3}}=0$, and $\mathcal{E} \mathcal{L}\left(\zeta^{2}\right)=\frac{1}{2}\left[\left(\frac{x}{\zeta}\right)^{2}+\right.$ $\left.\left(i \zeta \partial_{x}+\zeta^{\prime} x\right)^{2}\right]$ be the associated Ermakov-Lewis invariant.

Let $\phi \in \operatorname{Diff}_{+}(\mathbb{R} / 2 \pi \mathbb{Z})$ be a time-reparametrization and $\tilde{V}_{2}$ be the image of $V_{2}$ through $\phi$, defined by $\sigma_{1 / 4}(\phi) \cdot D=-2 i \partial_{\theta}-\partial_{r}^{2}+\tilde{V}_{2}(\theta) x^{2}$. 
Then:

(1) $\tilde{\zeta}:=\left(\phi^{\prime} \circ \phi^{-1}\right)^{1 / 2} \cdot \zeta \circ \phi^{-1}$ satisfies the transformed Pinney equation $\tilde{\zeta}^{\prime \prime}+$ $\tilde{V}_{2} \tilde{\zeta}-\frac{1}{\tilde{\zeta}^{3}}=0$.

(2) Consider the transformed Ermakov-Lewis invariant

$$
\widetilde{\mathcal{E} \mathcal{L}}\left(\tilde{\zeta}^{2}\right)(x):=\frac{1}{2}\left[\left(\frac{\tilde{x}}{\tilde{\zeta}}\right)^{2}+\left(i \tilde{\zeta} \partial_{\tilde{x}}+\frac{d \tilde{\zeta}}{d \tilde{\theta}} \tilde{x}\right)^{2}\right],
$$

where $(\tilde{\theta}, \tilde{x})=\left(\phi(\theta), x \sqrt{\phi^{\prime}(\theta)}\right)$ are the transformed coordinates.

Then

$$
\widetilde{\mathcal{E L}}\left(\tilde{\zeta}^{2}\right)=\pi_{1 / 4}(\phi) \mathcal{E} \mathcal{L}\left(\zeta^{2}\right) \pi_{1 / 4}(\phi)^{-1}
$$

In particular, $\widetilde{\mathcal{E} L}\left(\tilde{\zeta}^{2}\right)$ is an Ermakov-Lewis invariant for $\sigma_{1 / 4}(\phi) D$.

Proof. (1) follows from Lemma 3.1.5(2). This implies that $\widetilde{\mathcal{E L}}\left(\tilde{\zeta}^{2}\right)$ is an ErmakovLewis invariant for $\sigma_{1 / 4}(\phi) \cdot D$. Supposing one has proved that $\widetilde{\mathcal{E}}\left(\tilde{\zeta}^{2}\right)$ is the conjugate of $\mathcal{E} \mathcal{L}\left(\zeta^{2}\right)$ by $\pi_{1 / 4}(\phi)$, then it follows once again that $\widetilde{\mathcal{E} L}\left(\tilde{\zeta}^{2}\right)$ is an invariant for $\sigma_{1 / 4}(\phi) \cdot D$ since

$$
\begin{aligned}
& \left(\sigma_{1 / 4}(\phi) \cdot D\right) \widetilde{\mathcal{E} L}\left(\tilde{\zeta}^{2}\right)-\widetilde{\mathcal{E} \mathcal{L}}\left(\tilde{\zeta}^{2}\right)\left(\sigma_{1 / 4}(\phi) \cdot D\right) \\
& \quad=\phi^{\prime} \pi_{1 / 4}(\phi) D \cdot \mathcal{E} \mathcal{L}\left(\zeta^{2}\right) \cdot \pi_{1 / 4}(\phi)^{-1}-\pi_{1 / 4}(\phi) \cdot \mathcal{E} \mathcal{L}\left(\zeta^{2}\right) \cdot \phi^{\prime} D \pi_{1 / 4}(\phi)^{-1} \\
& \quad=0
\end{aligned}
$$

(the function of time $\phi^{\prime}$ commutes with the operator $\mathcal{E} \mathcal{L}\left(\zeta^{2}\right)$ ).

So all there remains to show is that $\widetilde{\mathcal{E} L}\left(\tilde{\zeta}^{2}\right)$ is indeed conjugate to $\mathcal{E} \mathcal{L}\left(\zeta^{2}\right)$. This is actually true for both terms appearing inside parentheses in the expression for the Ermakov-Lewis invariant (and trivial for the first one). Set $\mathcal{E}=i \zeta \partial_{x}+\zeta^{\prime} x$ and $\tilde{\mathcal{E}}=i \tilde{\zeta} \partial_{\tilde{x}}+\frac{d \tilde{\zeta}}{d \tilde{\theta}} \tilde{x}$. Then a simple computation shows that

$$
\tilde{\mathcal{E}}=i \zeta \partial_{x}+x \zeta^{\prime}+\frac{1}{2} x \frac{\phi^{\prime \prime}}{\phi^{\prime}} \zeta .
$$

On the other hand,

$$
\begin{aligned}
\left(\pi_{1 / 4}(\phi) \mathcal{E} \pi_{1 / 4}(\phi)^{-1}\right) \psi(\tilde{\theta}, \tilde{x}) \\
=\left(\phi^{\prime}(\theta)\right)^{-1 / 4} e^{\frac{1}{4} i \frac{\phi^{\prime \prime}(\theta)}{\phi^{\prime}(\theta)} x^{2}} \mathcal{E} \pi_{1 / 4}(\phi)^{-1} \psi(\theta, x) \\
=\frac{1}{2}\left(\phi^{\prime}(\theta)\right)^{-1 / 4} e^{\frac{1}{4} i \frac{\phi^{\prime \prime}(\theta)}{\phi^{\prime}(\theta)} x^{2}}\left(i \zeta(\theta) \partial_{x}\right. \\
\left.\quad+\zeta^{\prime}(\theta) x\right)\left(\phi^{\prime}(\theta)^{1 / 4} e^{-\frac{1}{4} i \frac{\phi^{\prime \prime}(\theta)}{\phi^{\prime}(\theta)} x^{2}} \psi\left(\phi(\theta), x \sqrt{\phi^{\prime}(\theta)}\right)\right) .
\end{aligned}
$$

Hence $\pi_{1 / 4}(\phi) \mathcal{E} \pi_{1 / 4}(\phi)^{-1}=\tilde{\mathcal{E}}$. 
We now want to be able to write the general solution of the Schrödinger equation as

$$
\psi(\theta)=\int_{\Sigma} e^{i \alpha_{k}(\theta)} c_{k} h_{k}(\theta) d \sigma(k)
$$

(for some spectral measure $\sigma$ on a set $\Sigma$, a discrete measure in the case studied by Lewis and Riesenfeld) with periodic eigenstates $h_{k}$ and a phase $\alpha_{k}$ with periodic derivative, i.e. given by integrating a periodic function, so that

$$
\psi(\theta+2 \pi)=\int e^{i \lambda_{k}} e^{i \alpha_{k}(\theta)} c_{k} h_{k}(\theta) d \sigma(k),
$$

where the $\lambda_{k}:=\alpha_{k}(\theta+2 \pi)-\alpha_{k}(\theta)$ are constants and measure the rotation of the eigenstates $h_{k}$ after a time $2 \pi$. Then the monodromy operator is unitarily equivalent to the multiplication operator $f(k) \rightarrow f(k) e^{i \lambda_{k}}$ on $L^{2}(\Sigma, d \sigma)$.

Consider any Schrödinger operator with quadratic potential $V_{2}(\theta) x^{2}$ and an associated nonzero vector field $\xi \in \operatorname{Stab}\left(V_{2}\right)$ as before. (We postpone the discussion of "resonant" operators (classes (i)bis and (iii)bis) to the next section.) It turns out that the eigenstates $h_{k}$ and the measure $\sigma$ can be taken as the (possibly generalized) eigenfunctions and spectral measure of one of the three following "model" operators $H$, depending on the sign of the invariant $I_{u}(\xi)$ :

(i) $\left(I_{u}(\xi)>0\right)$ : take for $H$ the standard harmonic oscillator

$$
H=-\frac{1}{2}\left(\partial_{x}^{2}-a^{2} x^{2}\right), \quad a \in \mathbb{R}
$$

this case corresponds to harmonic oscillators of type (i), i.e. Schrödinger operators of type (i) conjugate to $-2 i \partial_{\theta}-\partial_{x}^{2}+a^{2} x^{2}$ with $a^{2}>0$;

(ii) $\left(I_{u}(\xi)<0\right)$ : take for $H$ the "standard harmonic repulsor"

$$
H=-\frac{1}{2}\left(\partial_{x}^{2}+a^{2} x^{2}\right), \quad a \in \mathbb{R}
$$

this case corresponds to harmonic repulsors of type (i), i.e. operators of type (i) conjugate to $-2 i \partial_{\theta}-\partial_{x}^{2}-a^{2} x^{2}\left(-a^{2}<0\right)$, and operators of type (ii);

(iii) $\left(I_{u}(\xi)=0\right)$ : take for $H$ the usual one-dimensional Laplacian,

$$
H=-\frac{1}{2} \partial_{x}^{2}
$$

this case corresponds to operators of type (i) conjugate to the free Schrödinger operator $-2 i \partial_{\theta}-\partial_{x}^{2}$, and operators of type (iii).

Note that this classification is equivalent to the classification of the (conjugacy classes of) monodromy matrices for the associated Hill operators $\partial_{\theta}^{2}+V_{2}(\theta)$ into elliptic, hyperbolic and unipotent elements.

The next section circumvents the spectral analysis technicalities by solving the associated classical problem. The essentials for understanding the (operator-valued) monodromy for the quantum problem are already contained in the study of the $\left(\mathrm{SL}(2, \mathbb{R})\right.$-valued) monodromy of the ordinary differential equation $\ddot{x}=-V_{2}(\theta) x$, 
so we found this short digression convenient for the reader. Then we study the spectral decomposition of the above model operators. Finally, we solve the quantum problem for a quadratic potential $V_{2}(\theta) x^{2}$ and compute the monodromy operator. The general case $D \in \mathcal{S}_{\leq 2}^{\text {aff }}$ may be reduced to the quadratic case $D \in \mathcal{S}_{2}^{\text {aff }}$ after applying some transformation in SV, except for the operators of types (i)bis and (iii)bis which will be treated in the last section.

\subsection{Solution of the associated classical problem}

The associated classical problem (obtained for instance as the lowest-order term in $\hbar$ in the usual semiclassical expansion) is a Hill equation.

Definition 3.2.1. (classical problem) Let $H$ be the classical Hamiltonian

$$
H=\frac{1}{2}\left(p^{2}+V_{2}(\theta) x^{2}\right)
$$

The asociated motion in phase space reads $\dot{x}=\partial_{p} H=p, \dot{p}=-\partial_{x} H=-V_{2}$, which is equivalent to the Hill equation $\left(\partial_{\theta}^{2}+V_{2}\right) x(\theta)=0$.

Lemma 3.2.2. (1) Suppose $V_{2}$ is of type $I$ with $\alpha \neq 0$ or of type II, and choose $\xi \in \mathrm{StabV}_{2}$ so that $I_{u}(\xi)=2$ ( $\xi$ is real in the elliptic case and purely imaginary in the hyperbolic case). Then

$$
\mathcal{E} \mathcal{L}_{c l}(\xi)(x):=\frac{1}{2}\left[\frac{x^{2}}{\xi}+\xi\left(\dot{x}-\frac{1}{2} \frac{\dot{\xi}}{\xi} x\right)^{2}\right]
$$

is an invariant of the motion.

(2) Suppose $V_{2}$ is of type I with $\alpha=0$ or of type III (so that the associated monodromy is unipotent), and take any $\xi \in \mathrm{StabV}_{2}, \xi \neq 0$. Then

$$
\mathcal{E} \mathcal{L}_{c l}(\xi)(x):=\frac{1}{2}\left[\xi\left(\dot{x}-\frac{1}{2} \frac{\dot{\xi}}{\xi} x\right)^{2}\right]
$$

is an invariant of the motion.

Proof. Simple computation $\left(\mathcal{E} \mathcal{L}_{c l}\right.$ may be obtained from the quantum ErmakovLewis invariant by letting $\hbar$ go to zero).

Assuming $V_{2}$ is elliptic, i.e. of type I with $\alpha>0$, one may choose $\xi>0$. Then the equation $\mathcal{E} \mathcal{L}_{c l}(\xi)(x)=C, C$ constant is equivalent to $\left(\frac{d z}{d \tau}\right)^{2}+z^{2}=C$ after the function- and time-change $\tau(\theta)=\int^{\theta} \frac{d \theta^{\prime}}{\xi\left(\theta^{\prime}\right)}, x(\theta)=\xi^{1 / 2}(\theta) z(\tau(\theta))$, with obvious solutions $\cos \tau, \sin \tau$. Hence a basis of solutions of the equation of motion is 
given by

$$
x_{1}(\theta)=\xi^{1 / 2}(\theta) \cos \int^{\theta} \frac{d \theta^{\prime}}{\xi\left(\theta^{\prime}\right)}, \quad x_{2}(\theta)=\xi^{1 / 2}(\theta) \sin \int^{\theta} \frac{d \theta^{\prime}}{\xi\left(\theta^{\prime}\right)} .
$$

Assume for instance that $\dot{\xi}(0)=0$, and choose $\int^{\theta} \frac{d \theta^{\prime}}{\xi\left(\theta^{\prime}\right)}=\int_{0}^{\theta} \frac{d \theta^{\prime}}{\xi\left(\theta^{\prime}\right)}$. Then $\left(\begin{array}{l}x_{1} \\ x_{2}\end{array}\right)(2 \pi)=$ $\left(\begin{array}{cc}\cos T & -\sin T \\ \sin T & \cos T\end{array}\right) \cdot\left(\begin{array}{l}x_{1} \\ x_{2}\end{array}\right)(0)$ with $T=\int_{0}^{2 \pi} \frac{d \theta^{\prime}}{\xi\left(\theta^{\prime}\right)}$. Hence the eigenvalues of the monodromy matrix are given by $\pm i T$.

In the hyperbolic case (type I with $\alpha<0$, or type II), $\xi:=i \eta$ is purely imaginary. The above formulas (3.24) give solutions of the Hill equation on either side of any zero of $\xi$ (note that the normalization $I_{u}(\xi)=2$ implies $\xi(\theta) \sim_{\theta \rightarrow \theta_{0}} \pm 2 i\left(\theta-\theta_{0}\right.$ ) near any zero, so that (3.24) defines a continuous function, as should be), but the easiest way to define the solutions $x_{1}, x_{2}$ globally is to use a deformation of contour. One may always assume that $\xi$ is analytic on some complex neighborhood of $\mathbb{R}$ (it is conjugate by a time-reparametrization to some $u_{n, \alpha}$ which is entire, see Proposition 2.2.3). Define a contour $\Gamma$ from 0 to $2 \pi$ which avoids the zeros of $\xi$ by going around them along half-circles of small radii centered on the real axis. This time (see discussion in Sec. 2.2), the half-circles must be chosen alternatively in the upper- and lower-half planes so that $\operatorname{Re} \xi(z) \geq 0$ on $\Gamma$. Then $\left(\begin{array}{l}x_{1} \\ x_{2}\end{array}\right)(2 \pi)=$ $\left(\begin{array}{cc}\cos T & -\sin T \\ \sin T & \cos T\end{array}\right) \cdot\left(\begin{array}{l}x_{1} \\ x_{2}\end{array}\right)(0)$ as before, with $T=\int_{\Gamma} \frac{d \theta^{\prime}}{\xi\left(\theta^{\prime}\right)}$. Mind that $T$ is purely imaginary this time.

Finally, in the unipotent case (type I with $\alpha=0$, or type III), normalize $\xi$ by setting for instance $\xi(0)=i, \dot{\xi}(0)=0$, so that $\xi$ is purely imaginary. The same function- and time-change yields $\left(\frac{d z}{d \tau}\right)^{2}=C$, hence a natural basis of solutions is given by $x_{1}(\theta)=\xi^{1 / 2}(\theta), x_{2}(\theta)=\xi^{1 / 2}(\theta) \int_{0}^{\theta} \frac{d \theta^{\prime}}{\xi\left(\theta^{\prime}\right)}$. To get globally define solutions, one avoids the double zeros of $\xi$ by drawing half-circles in the upper half-plane. Then the monodromy matrix is $\left(\begin{array}{ll}1 & T \\ 0 & 1\end{array}\right)$, with $T=\int_{\Gamma} \frac{d \theta^{\prime}}{\xi\left(\theta^{\prime}\right)}=\int_{\Gamma} \frac{d \theta^{\prime}}{x_{1}^{2}\left(\theta^{\prime}\right)}$.

\subsection{Spectral decomposition of the model operators}

We shall need below the spectral decomposition of the three model operators $-\frac{1}{2}\left(\partial_{x}^{2}-a^{2} x^{2}\right),-\frac{1}{2}\left(\partial_{x}^{2}+a^{2} x^{2}\right),-\frac{1}{2} \partial_{x}^{2}$ introduced above. They are essentially selfadjoint on $C_{0}^{\infty}(\mathbb{R})$ by the classical Sears theorem (see [3], Theorem 1.1, Chap. 2 for instance), so the spectral theorem applies. The first operator has a pure point spectrum, while the second and third have an absolutely continuous spectrum. Note that $-\frac{1}{2} \partial_{x}^{2}$ is non-negative, while the spectrum of $-\frac{1}{2}\left(\partial_{x}^{2}+a^{2} x^{2}\right)$ is the whole real line, as the following lemma proves.

Lemma 3.3.1. (1) (elliptic case) The spectral decomposition of $L^{2}(\mathbb{R})$ for the operator $-\frac{1}{2}\left(\partial_{x}^{2}-a^{2} x^{2}\right)$ is given by

$$
L^{2}(\mathbb{R})=\oplus_{n \geq 0} L_{a\left(n+\frac{1}{2}\right)}^{2}
$$


where $L_{a\left(n+\frac{1}{2}\right)}^{2}$ is one-dimensional, generated by the normalized Hermite functions $\mathrm{Ca}^{1 / 4} e^{-a x^{2} / 2} \mathrm{He}_{n}(x \sqrt{a})$ for some constant $C$ (see [1] for the notations and normalization).

(2) (hyperbolic case) Set, for $\lambda \in \mathbb{R}$,

$$
\begin{aligned}
\psi_{\lambda}^{ \pm}(x):= & \left(\frac{2}{a}\right)^{1 / 4} e^{\lambda / 8 a} e^{-i a x^{2} / 2}\left[\frac{1}{\Gamma\left(\frac{3}{4}+\frac{i \lambda}{4 a}\right)}{ }_{1} F_{1}\left(\frac{1}{4}\left(1+\frac{i \lambda}{a}\right), \frac{1}{2} ; i a x^{2}\right)\right. \\
& \left. \pm \frac{2 \sqrt{a}}{\Gamma\left(\frac{1}{4}+\frac{i \lambda}{4 a}\right)} e^{i \pi / 4} x \cdot{ }_{1} F_{1}\left(\frac{1}{4}\left(3+\frac{i \lambda}{a}\right), \frac{3}{2} ; i a x^{2}\right)\right]
\end{aligned}
$$

where ${ }_{1} F_{1}$ is the usual confluent hypergeometric function. Then $H \psi_{\lambda}^{ \pm}=\lambda \psi_{\lambda}^{ \pm}$and the $\left(\psi_{\lambda}^{ \pm}, \lambda \in \mathbb{R}\right)$ form a complete orthonormal system of generalized eigenfunctions of the operator $H=-\frac{1}{2}\left(\partial_{x}^{2}+a^{2} x^{2}\right)$, so that any function $f \in L^{2}(\mathbb{R})$ decomposes uniquely as

$$
f(x)=\int_{\mathbb{R}} \psi_{\lambda}^{+}(x) \overline{g^{+}(\lambda)} d \lambda+\int_{\mathbb{R}} \psi_{\lambda}^{-}(x) \overline{g^{-}(\lambda)} d \lambda
$$

with $g^{ \pm}(\lambda)=\int_{\mathbb{R}} f(x) \overline{\psi_{\lambda}^{ \pm}(x)} d x$. In particular, the following Parseval identity holds,

$$
\int_{\mathbb{R}}|f(x)|^{2} d x=\int_{\mathbb{R}}\left|g^{+}(\lambda)\right|^{2} d \lambda+\int_{\mathbb{R}}\left|g^{-}(\lambda)\right|^{2} d \lambda .
$$

(3) (unipotent case) Set, for $\lambda>0, \psi_{\lambda}^{ \pm}(x)=e^{ \pm i x \sqrt{2 \lambda}}$. Then $H \psi_{\lambda}^{ \pm}=\lambda \psi_{\lambda}^{ \pm}$and the $\psi_{\lambda}^{ \pm}, \lambda>0$, form a complete orthonormal system of generalized eigenfunctions of the operator $H=-\frac{1}{2} \partial_{x}^{2}$, with the usual Parseval-Bessel identity.

Proof. (1) is classical and (3) is straightforward by Fourier inversion and the usual Parseval-Bessel identity. Case (2) is less common, though it can certainly be found somewhere in the literature. Let us explain briefly how to obtain its spectral decomposition for $a=1$. The easiest way is to remark that $H=A \Lambda A^{-1}$ where $\Lambda=\frac{i}{2}\left(x \partial_{x}+\partial_{x} x\right)=i\left(x \partial_{x}+\frac{1}{2}\right)$ and $A$ is the image of the rotation matrix $\left(\begin{array}{cc}\cos \pi / 4 & -\sin \pi / 4 \\ \sin \pi / 4 & \cos \pi / 4\end{array}\right)$ by the metaplectic representation. The operator $A$ is unitary. Explicit formulas found for instance in [15] show that

$$
(A f)(x)=i \sqrt{2} e^{i \pi / 4} e^{i \pi x^{2}} \int_{0}^{\infty} e^{-i \pi(x \sqrt{2}-y)^{2}} f(y) d y .
$$

As for the operator $\Lambda$, it is conjugate to $i\left(\partial_{y}+\frac{1}{2}\right)$ after the obvious change of variable $x= \pm e^{y}$, hence its spectral decomposition is given by Fourier inversion on either half-lines, $\Lambda \phi_{\lambda}^{ \pm}=\lambda \phi_{\lambda}^{ \pm}(\lambda \in \mathbb{R})$ with $\phi_{\lambda}^{ \pm}(x)=x_{ \pm}^{-\frac{1}{2}-i \lambda}$ constituting an orthonormal basis of generalized eigenfunctions. Finally, $\psi_{\lambda}^{ \pm}:=A \phi_{\lambda}^{ \pm}$may be obtained by 
applying the following formula (see [10])

$$
\int_{0}^{\infty} x^{\nu-1} e^{-\beta x^{2}-\gamma x} d x=(2 \beta)^{-\nu / 2} \Gamma(\nu) e^{\gamma^{2} / 8 \beta} D_{-\nu}\left(\frac{\gamma}{\sqrt{2 \beta}}\right)
$$

$(\operatorname{Re} \beta, \operatorname{Re} \nu>0)$ where $D_{\nu}$ is a parabolic cylinder function, also given by

$$
\begin{aligned}
D_{\nu}(z)= & 2^{\nu / 2} e^{-z^{2} / 4}\left\{\frac{\sqrt{\pi}}{\Gamma\left(\frac{1}{2}(1-\nu)\right)}{ }_{1} F_{1}\left(-\frac{\nu}{2}, \frac{1}{2} ; z^{2} / 2\right)\right. \\
& \left.-z \frac{\sqrt{2 \pi}}{\Gamma(-\nu / 2)}{ }_{1} F_{1}\left(\frac{1-\nu}{2}, \frac{3}{2} ; z^{2} / 2\right)\right\}
\end{aligned}
$$

(see [7], 8.2. (4), p. 117).

\subsection{Monodromy of non-resonant harmonic oscillators (elliptic case)}

We assume here that $D \in \mathcal{S}_{\leq 2}^{\text {aff }}$ is of class (i) with $\alpha>0$. Then $D$ is conjugate by a transformation in SV to an operator of the type $-2 i \partial_{\theta}-\partial_{x}^{2}+a^{2} x^{2}+\gamma$ where $a>0$ and $\gamma$ is a constant. Choose $\xi=\frac{1}{a}$ so that $\sqrt{\xi}$ satisfies a normalized Pinney-Milne equation. Then Proposition 3.1.4 shows the following:

Theorem 3.4.1. The solution of the Schrödinger equation with arbitrary initial state

$$
\psi(0):=\sum_{n \geq 0} c_{n} h_{n}(0)
$$

is given by

$$
\psi(\theta):=\sum_{n \geq 0} c_{n} e^{-i\left(n+\frac{1}{2}\right) a \theta-i \gamma \theta / 2} h_{n}(\theta)
$$

The monodromy operator is given by the "infinite-dimensional" monodromy matrix $M_{D}:=\operatorname{diag}\left(e^{i \lambda_{n}}, n \in \mathbb{N}\right)$, with $\lambda_{n}:=-2 \pi\left(n+\frac{1}{2}\right) a-\pi \gamma$.

\subsection{Monodromy of harmonic repulsors (hyperbolic type)}

One assumes now that $D \in \mathcal{S}_{\leq 2}^{\text {aff }}$ is either of class (i) with $\alpha<0$ or of class (ii). Consider again the Ermakov-Lewis invariant

$$
\mathcal{E} \mathcal{L}(\xi)=\frac{1}{2 \xi}\left[x^{2}+\left(i \xi \partial_{x}+\frac{1}{2} \dot{\xi} x\right)^{2}\right],
$$

where one has assumed that $\xi=i \eta$ is purely imaginary this time, and $I_{V_{2}}(\xi)=2$. Note that $\mathcal{E} \mathcal{L}(i \eta)$ is anti-hermitian. Then

$$
\frac{\mathcal{E} \mathcal{L}(i \eta)-i k}{i \eta}=-\frac{1}{2}\left[\partial_{x}^{2}-i \frac{\dot{\eta}}{\eta} x \partial_{x}+\frac{1-\frac{1}{4} \dot{\eta}^{2}}{\eta^{2}} x^{2}-\frac{1}{2} i \frac{\dot{\eta}}{\eta}+\frac{2 k}{\eta}\right] .
$$


Suppose $\psi_{k} \neq 0$ is an eigenvector of the Ermakov-Lewis operator, $\mathcal{E} \mathcal{L}(i \eta) \psi_{k}=$ $i k \psi_{k}$. Then Proposition 2.1.4 implies that $\tilde{\psi}_{k}:=\exp -\frac{i}{4} \frac{\dot{\eta}}{\eta} x^{2} \cdot \psi_{k}$ is a generalized eigenfunction of the model harmonic repulsor, namely

$$
-\frac{1}{2}\left(\partial_{x}^{2}+\frac{x^{2}}{\eta^{2}}\right) \tilde{\psi}_{k}=\frac{k}{\eta} \tilde{\psi}_{k} .
$$

Hence:

Lemma 3.5.1. (1) The equation $(\mathcal{E} \mathcal{L}(i \eta)-i k) \psi_{k}=0(k \in \mathbb{R})$ has two linearly independent solutions,

$$
\psi_{\text {even }}^{k}(\theta, x)=\sqrt{2}(2 i \eta)^{1 / 4} e^{k / 4} e^{\frac{i}{4} \frac{\dot{\eta}}{\eta} x^{2}} e^{-\frac{i}{2 \eta} x^{2}} \cdot \frac{1}{\Gamma\left(\frac{1}{4}+i \frac{k}{2}\right)}{ }_{1} F_{1}\left(\frac{1}{4}(1+2 i k), \frac{1}{2} ; \frac{i x^{2}}{\eta}\right)
$$

and

$$
\psi_{\text {odd }}^{k}(\theta, x)=2 \sqrt{2}(2 i \eta)^{1 / 4} e^{k / 4} e^{\frac{i}{4} \frac{\dot{\eta}}{\eta} x^{2}} e^{-\frac{i}{2 \eta} x^{2}} \frac{1}{\Gamma\left(\frac{3}{4}+i \frac{k}{2}\right)} x \cdot{ }_{1} F_{1}\left(\frac{1}{4}(3+2 i k), \frac{3}{2} ; \frac{i x^{2}}{\eta}\right) .
$$

The functions $\left(\left(\psi_{\text {even }}^{k}, \psi_{\text {odd }}^{k}\right), k \in \mathbb{R}\right)$ constitute a complete orthonormal system for the operator $\mathcal{E} \mathcal{L}(i \eta)$.

(2) One has

$$
D \psi_{\mathrm{even}}^{k}(x)=\left(\frac{2 k}{\eta}-i \frac{\dot{\eta}}{\eta}\right) \psi_{\mathrm{even}}^{k}(x)
$$

and

$$
D \psi_{\text {odd }}^{k}(x)=\left(\frac{2 k}{\eta}-2 i \frac{\dot{\eta}}{\eta}\right) \psi_{\text {odd }}^{k}(x) .
$$

Hence $x \rightarrow \frac{1}{\sqrt{\xi}} \exp \left(k \int^{\theta} \frac{d \theta^{\prime}}{\xi\left(\theta^{\prime}\right)}\right) \psi_{\text {even }}^{k}(x)$ and $x \rightarrow \frac{1}{\xi} \exp \left(k \int^{\theta} \frac{d \theta^{\prime}}{\xi\left(\theta^{\prime}\right)}\right) \psi_{\text {odd }}^{k}(x)$ are solutions of the Schrödinger equation.

Proof. (1) is a direct application of Lemma 3.3.1, while (2) follows from an easy computation using the confluent hypergeometric differential equation

$$
z \frac{d^{2}}{d z^{2}}{ }_{1} F_{1}(a, c ; z)+(c-z) \frac{d}{d z}{ }_{1} F_{1}(a, c, ; z)-a_{1} F_{1}(a, c ; z)=0 .
$$

The eigenfunctions $\psi_{\text {even }}^{k}, \psi_{\text {odd }}^{k}$ depend analytically on $\xi$ for $\xi \in \mathbb{C} \backslash \mathbb{R}$. . If the operator $D$ is of type I (so that $\xi$ has no zero), say with $\gamma=0$, then the phase $\exp \left(k \int^{\theta} \frac{d \theta^{\prime}}{\xi\left(\theta^{\prime}\right)}\right)$ gives the monodromy. If $D$ is of type II, then one must resort to a deformation of contour in order to avoid the singularities, as in the classical case, see Sec. 3.2. Note that the deformation of contour may change drastically the behavior of the functions $\psi_{\text {even }}^{k}, \psi_{\text {odd }}^{k}$ for large $x$ or large $k$ (for instance, $\psi_{\text {even }}^{k}$ and $\psi_{\text {odd }}^{k}$ become exponentially increasing for large $x$ ). Hence, in order to be able to follow the phase shift of the eigenfunctions $\psi_{\text {even }}^{k}, \psi_{\text {odd }}^{k}$ along the contour $\Gamma$ without getting divergent integrals, it is better to assume to begin with that the "Fourier transform" (with respect to the spectral decomposition of $\mathcal{E} \mathcal{L}(\xi)$ ) of the solution 
has compact support. In other words, the solution of the Schrödinger equation with initial state

$$
\psi(0, x):=\int_{\mathbb{R}} \bar{c}_{+}(k) \psi_{\text {even }}^{k}(0, x) d k+\int_{\mathbb{R}} \bar{c}_{-}(k) \psi_{\text {odd }}^{k}(0, x) d k
$$

for $z \in \Gamma$ (complex time), where $c_{+}, c_{-}$are assumed to be compactly supported, is given by

$$
\begin{aligned}
\psi(z, x)= & \sqrt{\frac{\xi(0)}{\xi(z)}} \int_{\mathbb{R}} \bar{c}_{+}(k) e^{k \int_{0}^{z} \frac{d z^{\prime}}{\xi\left(z^{\prime}\right)}-i \gamma \theta / 2} \psi_{\text {even }}^{k}(z, x), d k \\
& +\frac{\xi(0)}{\xi(z)} \int_{\mathbb{R}} \bar{c}_{-}(k) e^{k \int_{0}^{z} \frac{d z^{\prime}}{\xi\left(z^{\prime}\right)}-i \gamma \theta / 2} \psi_{\text {odd }}^{k}(z, x) d k
\end{aligned}
$$

An immediate corollary is:

Theorem 3.5.2. Let $\psi(0) \in L^{2}(\mathbb{R})$, with decomposition

$$
\psi(0, x):=\int_{\mathbb{R}} \bar{c}_{+}(k) \psi_{\text {even }}^{k}(0, x) d k+\int_{\mathbb{R}} \bar{c}_{-}(k) \psi_{\text {odd }}^{k}(0, x) d k .
$$

Then the solution of any type (ii) Schrödinger equation with initial state $\psi(0)$ is given at time $\theta=2 \pi$ by

$$
\psi(2 \pi, x)=\int_{\mathbb{R}} \bar{c}_{+}(k) e^{k T-i \pi \gamma} \psi_{\text {even }}^{k}(0, x) d k+\int_{\mathbb{R}} \bar{c}_{-}(k) e^{k T-i \pi \gamma} \psi_{\text {odd }}^{k}(0, x) d k,
$$

where $T=\int_{0}^{2 \pi} \frac{d u}{\xi(u)}$ or $\int_{\Gamma} \frac{d u}{\xi(u)}$ (depending on the class of $V_{2}$ ), with $\Gamma$ chosen as in Sec. 3.2, is purely imaginary. The associated monodromy operator in $\mathcal{B}\left(L^{2}(\mathbb{R}), L^{2}(\mathbb{R})\right)$ is unitarily equivalent to the multiplication by the function $k \rightarrow$ $e^{k T-i \pi \gamma}$ with modulus one.

\subsection{Monodromy of non-resonant operators of unipotent type}

Suppose now $D \in \mathcal{S}_{\leq 2}^{\text {aff }}$ is of class (i), $\alpha=0$ or (iii). Then

$$
\mathcal{E} \mathcal{L}(\xi)(\theta):=\frac{1}{2 \xi}\left[\left(i \xi \partial_{x}+\frac{1}{2} \dot{\xi} x\right)^{2}\right]
$$

$\left(\xi \in \mathrm{Stab}_{V_{2}}\right.$ ) is an invariant of $D$ (note the difference with respect to Proposition 3.1.4). Case (i), $\alpha=0$ is trivial, for it is conjugate to the free Schrödinger equation. So assume $D=-2 i \partial_{\theta}-\partial_{x}^{2}+V_{2} x^{2}$ is of class III. Take $\xi=i \eta$ with $\eta \geq 0$ as in Sec. 3.2. Then (if $k>0$ )

$$
\frac{\mathcal{E} \mathcal{L}(\xi)-i k}{\xi}=-\frac{1}{2}\left(\partial_{x}-\frac{i}{2} \frac{\dot{\eta}}{\eta} x\right)^{2}-\frac{k}{\eta}
$$

So

$$
\psi_{k, \pm}(x):=\exp \frac{i}{4} \frac{\dot{\eta}}{\eta} x^{2} \cdot \exp \pm i \sqrt{\frac{2 k}{\eta}} x
$$

constitute a complete orthonormal system for $\mathcal{E} \mathcal{L}(i \eta)$ (the same statement holds true for potentials of class (i), in which case $\eta=1$ and the exponential prefactor is 
trivial). A short computation shows that

$$
D \psi_{k, \pm}=\left(\frac{2 k}{\eta}-\frac{i}{2} \frac{\dot{\eta}}{\eta}\right) \psi_{k, \pm}
$$

Hence one has the following:

Theorem 3.6.1. Let $\psi(0) \in L^{2}(\mathbb{R})$, with decomposition

$$
\psi(0, x):=\int_{\mathbb{R}_{+}} \bar{c}_{+}(k) \psi_{k,+}(x) d k+\int_{\mathbb{R}_{+}} \bar{c}_{-}(k) \psi_{k,-}(x) d k .
$$

Then the solution of any type (i), $\alpha=0$ or type (iii) Schrödinger equation with initial state $\psi(0)$ is given at time $\theta=2 \pi$ by

$$
\psi(2 \pi, x)=\int_{\mathbb{R}_{+}} \bar{c}_{+}(k) e^{k T-i \pi \gamma} \psi_{k,+}(x) d k+\int_{\mathbb{R}_{+}} \bar{c}_{-}(k) e^{k T-i \pi \gamma} \psi_{k,-}(x) d k,
$$

where $T=\int_{0}^{2 \pi} \frac{d u}{\xi(u)}$ or $\int_{\Gamma} \frac{d u}{\xi(u)}, \Gamma$ chosen as in Sec. 3.2 (depending on the class of $\left.V_{2}\right)$ is purely imaginary. The associated monodromy operator in $\mathcal{B}\left(L^{2}(\mathbb{R}), L^{2}(\mathbb{R})\right)$ is unitarily equivalent to the unitary operator on $L^{2}\left(\mathbb{R}_{+}\right)$given by the multiplication by the function $k \rightarrow e^{k T-i \pi \gamma}$.

\section{Symplectic Structures and General Solution of the Schrödinger Equation}

The general emphasis in this section is so to speak on the non-quadratic part of the potential, namely, on $V_{0}$ and $V_{1}$ if $D=-2 i \partial_{\theta}-\partial_{x}^{2}+V_{2}(\theta) x^{2}+V_{1}(\theta) x+$ $V_{0}(\theta)$. It contains somewhat loosely related results: a definition of a threedimensional invariant $\left(\xi, \delta_{1}, \delta_{2}\right)$; a generalization of the Ermakov-Lewis invariants to general potentials; a symplectic structure on a space "containing" $\mathcal{S}_{\leq 2}^{\text {aff }}$ such that the SV-action becomes naturally Hamiltonian; finally, the computation of the monodromy for the "resonant" operators of type (i)bis, (iii)bis.

Definition 4.1. We shall say that $D \in \mathcal{S}_{\leq 2}^{\text {aff }}$ is of generic type if: $D$ is of class (i), $D$ conjugate to $D_{\alpha, \gamma}=-2 i \partial_{\theta}-\partial_{x}^{2}+\alpha x^{2}+\gamma$ with $\alpha \neq n^{2} / 4, n=0,1, \ldots$; or $D$ is of class (ii), $D$ conjugate to $D_{n, \alpha, \gamma}=-2 i \partial_{\theta}-\partial_{x}^{2}+u_{n, \alpha}(\theta) x^{2}+\gamma$.

Denote by $\mathcal{S}_{\leq 2}^{\text {aff } \text {, gen }}$ the set of operators of generic type; it is a disjoint union of SV-orbits.

Note (see Corollary 2.4.4) that the isotropy group of an operator $D$ of generic type is generated by $\mathcal{M}_{1}$ and some $\mathcal{L}_{\xi}+\mathcal{Y}_{f_{1}}+\mathcal{M}_{f_{2}}$ with $\xi \neq 0$.

Definition 4.2. Let $D=-2 i \partial_{\theta}-\partial_{x}^{2}+V_{2}(\theta) x^{2}+V_{1}(\theta) x+V_{0}(\theta) \in \mathcal{S}_{\leq 2 \text {,gen }}^{\text {aff }}$ be of generic type.

Define:

(i) $\xi(D)$ to be the unique (up to a sign) periodic vector field such that $\xi(D) \in$ $\mathrm{Stab}_{V_{2}}$ and $I_{V_{2}}(\xi(D))=2$ ( $\xi$ real in the elliptic case, purely imaginary in the hyperbolic case); 
(ii) $\delta_{1}(D)$ to be the unique periodic function such that

$$
\ddot{\delta_{1}}(D)+V_{2} \delta_{1}(D)=-\frac{1}{2}\left(\dot{V}_{1} \xi(D)+\frac{3}{2} V_{1} \dot{\xi}(D)\right) ;
$$

(iii) $\delta_{2}(D)$ to be the unique periodic function (up to a constant) such that

$$
\delta_{2}(D)=-\frac{1}{2} \int^{\theta} V_{1}\left(\theta^{\prime}\right) \delta_{1}(D)\left(\theta^{\prime}\right) d \theta^{\prime}-\frac{1}{2} V_{0} \xi(D) .
$$

Observe that $\mathcal{L}_{\xi}+\mathcal{Y}_{\delta_{1}}+\mathcal{M}_{\delta_{2}} \in \operatorname{Lie}\left(G_{D}\right)$ is indeed unique (up to the addition of a constant times $\mathcal{M}_{1}$ ) as follows from Corollary 2.4.4. The ambiguity in the definition of $\delta_{2}$ may be solved by choosing for each SV-orbit an arbitrary base-point, an invariant $\left(\xi, \delta_{1}, \delta_{2}\right)$ for this base-point, and transforming $\left(\xi, \delta_{1}, \delta_{2}\right)$ covariantly by the adjoint action along the orbit. Some nonlocal formulas fixing $\delta_{2}$ more explicitly can probably be found, at least for potentials of type (i) (see Lemma 2.4.5), but we shall not need them.

Another problem comes from the fact that the map $\left(V_{2}, V_{1}, V_{0}\right) \rightarrow\left(\xi, \delta_{1}, \delta_{2}\right)$ is not one-to-one (nor onto). Suppose one has some triple of functions $\left(\xi, \delta_{1}, \delta_{2}\right)$. Under some conditions that we shall not write explicitly (depending on the class of the potential), $\left(\xi, \delta_{1}, \delta_{2}\right)$ is an invariant for some potential $\left(V_{2}, V_{1}, V_{0}\right)$; the quadratic part $V_{2}$ is given (by definition) by $V_{2}=\frac{1}{2 \xi^{2}}\left(2-\xi \ddot{\xi}+\frac{1}{2} \dot{\xi}^{2}\right.$ ). (Supposing $\xi$ has only a finite number of zeros, all of which are simple or double, one has some rather straightforward conditions on the values of $\frac{d \xi}{d \theta}$ and $\frac{d^{3} \xi}{d \theta^{3}}$ at the zeros of $\xi$ that ensure that $\xi \in \operatorname{Stab}_{V_{2}}$ for some potential $V_{2}$.) But $V_{1}$ is not determined uniquely if $\xi$ does not vanish on the torus, since $\xi^{-3 / 2}$ is in the kernel of the operator $\xi \partial+\frac{3}{2} \dot{\xi}$ (see formula (4.1)). This can easily be explained by supposing (by conjugating by some element $g \in S V$ ) that $D$ is the model operator $D=-2 i \partial_{\theta}-\partial_{x}^{2}+\alpha x^{2}+\gamma$ ( $\alpha$ generic). Then $\xi$ is proportional to the constant vector field $\mathcal{L}_{1}$ which commutes with $\mathcal{Y}_{1}$, hence the invariant $\left(\xi, \delta_{1}, \delta_{2}\right)$ is left unchanged by space-translations, whereas the operator $D$ (and also the generalized Ermakov-Lewis invariant defined in Theorem 4.4 below) is not. Hence the vector invariant $\left(\xi, \delta_{1}, \delta_{2}\right)$ parametrizes Schrödinger operators of type (i) "up to space-translations". On the other hand, the map $\left(V_{2}, V_{1}, V_{0}\right) \rightarrow\left(\xi, \delta_{1}, \delta_{2}\right)$ is one-to-one for operators of type (ii) (up to a sign for $\xi)$.

It is not a priori self-evident that $\delta_{2}$ defined by Eq. (4.2) is a periodic function. Considering the "inverse problem", i.e. supposing that the invariant $\left(\xi, \delta_{1}, \delta_{2}\right)$ is given, and supposing $\xi$ does not vanish on the torus, one must also check that every choice for $V_{1}$ gives a function $\delta_{2}$ which is periodic. This is the content of the following lemma:

Lemma 4.3. One has:

$$
\frac{d}{d \theta}\left(\xi \frac{d}{d \theta}\left(\xi^{-\frac{1}{2}} \delta_{1}\right)\right)=-\frac{1}{2} \frac{d}{d \theta}\left(\xi^{3 / 2} V_{1}\right)-\xi^{-3 / 2} \delta_{1}
$$

This formula implies: $\int_{0}^{2 \pi} \xi^{-3 / 2} \delta_{1}=0 ; \int_{0}^{2 \pi} V_{1} \delta_{1}=0$. 
Proof. Using the invariant equations $\xi \ddot{\xi}-\frac{1}{2} \dot{\xi}^{2}+2 V_{2} \xi^{2}=2$ and $\ddot{\delta}_{1}+V_{2} \delta_{1}=$ $-\frac{1}{2}\left(\dot{V}_{1} \xi+\frac{3}{2} V_{1} \dot{\xi}\right)$, one obtains

$$
\frac{d}{d \theta}\left(\xi^{1 / 2} \dot{\delta}_{1}\right)=\frac{1}{2} \frac{d}{d \theta}\left(\xi^{-\frac{1}{2}} \dot{\xi} \delta_{1}\right)-\frac{1}{2} \frac{d}{d \theta}\left(\xi^{3 / 2} V_{1}\right)-\xi^{-3 / 2} \delta_{1}
$$

hence the first equation, which implies immediately: $\int_{0}^{2 \pi} \xi^{-3 / 2}(\theta) \delta_{1}(\theta) d \theta=0$. Hence (considering the inverse problem), if some potential $V_{1}$ verifies $\int_{0}^{2 \pi} V_{1}(\theta) \delta_{1}(\theta) d \theta=0$ (so that $\delta_{2}$ is well-defined), then this is also true for all possible potentials $V_{1}$. Now, integrating the first equation, one gets

$$
\xi \frac{d}{d \theta}\left(\xi^{-\frac{1}{2}} \delta_{1}\right)+\frac{1}{2} \xi^{3 / 2} V_{1}=-\int^{\theta} \xi^{-3 / 2}\left(\theta^{\prime}\right) \delta_{1}\left(\theta^{\prime}\right) d \theta^{\prime},
$$

hence

$$
\xi^{1 / 2} V_{1}=-2\left[\frac{d}{d \theta}\left(\xi^{-\frac{1}{2}} \delta_{1}\right)+\frac{1}{\xi} \int^{\theta} \xi^{-3 / 2}\left(\theta^{\prime}\right) \delta_{1}\left(\theta^{\prime}\right) d \theta^{\prime}\right]
$$

Hence

$$
\begin{aligned}
\int^{\theta} V_{1}\left(\theta^{\prime}\right) \delta_{1}\left(\theta^{\prime}\right) d \theta^{\prime} & =\int^{\theta}\left(\xi^{1 / 2} V_{1}\right)\left(\theta^{\prime}\right)\left(\xi^{-\frac{1}{2}} \delta_{1}\right)\left(\theta^{\prime}\right) d \theta^{\prime} \\
& =-\left[\left(\xi^{-\frac{1}{2}}(\theta) \delta_{1}(\theta)\right)^{2}+\left(\int^{\theta} \xi^{-3 / 2}\left(\theta^{\prime}\right) \delta_{1}\left(\theta^{\prime}\right) d \theta^{\prime}\right)^{2}\right]
\end{aligned}
$$

and the integral over a period is zero.

The following covariance result is an extension of Theorem 3.1.6.

Theorem 4.4. Let $D \in \mathcal{S}_{\leq 2 \text {,gen }}^{\text {aff }}$ be of generic type, with associated invariant $(\xi=$ $\left.\xi(D), \delta_{1}=\delta_{1}(D), \delta_{2}=\delta_{2}(D)\right)$. Then

$$
\begin{aligned}
\mathcal{E} \mathcal{L}(D):= & \frac{1}{2}\left[\frac{1}{\xi}\left(1+\frac{1}{4} \dot{\xi}^{2}\right) x^{2}-\xi \partial_{x}^{2}+\frac{i}{2} \dot{\xi}\left(x \partial_{x}+\partial_{x} x\right)+\left(-2 \delta_{1}\left(-i \partial_{x}\right)\right.\right. \\
& \left.\left.+\left(V_{1} \xi+2 \dot{\delta_{1}}\right) x\right)+2\left(\delta_{2}+\frac{1}{2} V_{0} \xi\right)\right]
\end{aligned}
$$

is an invariant for the Schrödinger operator $D$.

(2) Let $(\phi ;(a, b)) \in \mathrm{SV}$ and $g:(\theta, x) \rightarrow\left(\theta^{\prime}, x^{\prime}\right)=(\phi(\theta), x \sqrt{\dot{\phi}(\theta)}-a(\theta))$ be the associated coordinate change. Then

$$
\pi_{1 / 4}(\phi ;(a, b)) \mathcal{E} \mathcal{L}(D) \pi_{1 / 4}(\phi ;(a, b))^{-1}=\widetilde{\mathcal{E L}}(D)
$$

where $\widetilde{\mathcal{E} L}(D)$ is obtained by applying the transformation $g$ to the coordinates, changing the potentials $V_{0}$ and $V_{1}$ by the $\sigma_{1 / 4}$-action of $S V$, and transforming the invariant 
as follows:

$$
\begin{gathered}
\tilde{\xi}=\phi^{\prime} \cdot \xi \circ \phi^{-1} \\
\tilde{\delta_{1}}=\phi^{1 / 2} \cdot \delta_{1} \circ \phi^{-1}+\left(\tilde{\xi} \dot{a}-\frac{1}{2} a \dot{\tilde{\xi}}\right) \\
\tilde{\delta_{2}}=\delta_{2} \circ \phi^{-1}+\left(\delta_{1} \dot{a}-a \dot{\delta_{1}}\right)+\tilde{\xi} \dot{b}+\left(\tilde{\xi}\left(\dot{a}^{2}-a \ddot{a}\right)-\dot{\tilde{\xi}} a \dot{a}-\ddot{\tilde{\xi}} a^{2}\right) .
\end{gathered}
$$

Furthermore, $\left(\tilde{\xi}, \tilde{\delta}_{1}, \tilde{\delta}_{2}\right)$ is the invariant associated to $\sigma_{1 / 4}(D)$.

Proof. (1) Look for an invariant of the form

$$
\frac{1}{2}\left[a(\theta) x^{2}-b(\theta) \partial_{x}^{2}-i c(\theta)\left(x \partial_{x}+\partial_{x} x\right)+d(\theta)\left(-i \partial_{x}\right)+e(\theta) x+f(\theta)\right]
$$

and solve in $a, b, c, d, e, f$. One obtains the following constraints:

$$
\dot{a}=2 V_{2} c, \quad \dot{b}=-2 c, \quad \dot{c}=-a+V_{2} b,
$$

whose general solution is in Proposition 3.1.4 above, namely, $a=\frac{1}{\xi}\left(1+\frac{1}{4} \dot{\xi}^{2}\right), b=\xi$, $c=-\frac{1}{2} \dot{\xi}$, and the set of following equations:

$$
\dot{d}=V_{1} b-e, \quad \dot{e}=V_{1} c+V_{2} d, \quad \dot{f}=\frac{1}{2} d V_{1}
$$

which implies the compatibility condition

$$
\ddot{d}+V_{2} d=\dot{V}_{1} \xi+\frac{3}{2} V_{1} \dot{\xi}
$$

(2) Since (assuming $I_{u}(\xi)=2$ is fixed), there is a unique invariant for operators of generic type, one necessarily has

$$
\mathcal{L}_{\tilde{\xi}}+\mathcal{Y}_{\tilde{\delta_{1}}}+\mathcal{M}_{\tilde{\delta_{2}}}=\operatorname{Ad}(\phi ;(a, b)) \cdot\left(\mathcal{L}_{\xi}+\mathcal{Y}_{\delta_{1}}+\mathcal{M}_{\delta_{2}}\right)
$$

which gives the above formulas for $\left(\tilde{\xi}, \tilde{\delta_{1}}, \tilde{\delta_{2}}\right)$.

There remains to check for Eq. (4.6). Consider first the covariance under a time-reparametrization $\phi$. It has already been proved for the quadratic part of the Ermakov-Lewis operator, see Theorem 3.1.6. The linear part $-2\left(-i \delta_{1} \partial_{x}+\left(V_{1} \xi-\right.\right.$ $\left.\dot{\delta_{1}}\right) x$ ) transforms covariantly under $\phi$ since (see proof of Theorem 3.1.6)

$$
\tilde{V}_{1} \tilde{\xi}-\frac{d \tilde{\delta_{1}}}{d \theta^{\prime}}=\phi^{\prime-\frac{1}{2}}\left(V_{1} \cdot \xi-\dot{\delta_{1}}-\frac{1}{2} \frac{\ddot{\phi}}{\dot{\phi}} \delta_{1}\right), \quad-i \tilde{\delta_{1}} \partial_{x^{\prime}}=-i \delta_{1} \partial_{x}
$$

and

$$
\begin{aligned}
& \left(\pi_{1 / 4}(\phi)\left(-i \delta_{1}(\theta) \partial_{x}+\left(V_{1} \xi-\dot{\delta}_{1}\right) x\right) \pi_{1 / 4}\left(\phi^{-1}\right)\right) \psi \\
& \quad=\frac{1}{2} \dot{\phi}^{-1 / 4} e^{\frac{i}{4} \frac{\ddot{\phi}}{\phi} x^{2}}\left(-i \delta_{1} \partial_{x}+\left(V_{1} \xi-\dot{\delta}_{1}\right) x\right) \dot{\phi}^{1 / 4} e^{-\frac{i}{4} \frac{\ddot{\phi}}{\phi} x^{2}} \psi(\phi(\theta), x \sqrt{\dot{\phi}(\theta)}) \\
& \quad=\left(-i \tilde{\delta}_{1} \partial_{x^{\prime}}+\left(\tilde{V}_{1} \tilde{\xi}-\tilde{\delta}_{1}\right) x^{\prime}\right) \psi\left(\theta^{\prime}, x^{\prime}\right) .
\end{aligned}
$$


As for the zero-order term $-\frac{1}{2}\left(\delta_{2}+\frac{1}{2} V_{0} \xi\right)$, it is obviously invariant under the conjugate action of $\pi_{1 / 4}(\phi)$. Since $\tilde{V}_{0} \tilde{\xi}=\left(V_{0} \xi\right) \circ \phi^{-1}$, this implies also $\tilde{\delta}_{2}=\delta_{2} \circ \phi^{-1}$.

Consider now the covariance under an infinitesimal nilpotent transformation $\mathcal{Y}_{f_{1}}+\mathcal{M}_{f_{2}}$. One has

$$
\begin{aligned}
{\left[a \partial_{x}\right.} & \left.+i \dot{a} x+b, \mathcal{E} \mathcal{L}\left(\xi, \delta_{1}, \delta_{2}\right)\right] \\
& =\frac{1}{2}\left[a \partial_{x}+i \dot{a} x, \frac{1}{\xi}\left(1+\frac{1}{4} \dot{\xi}^{2}\right) x^{2}-\xi \partial_{x}^{2}+i \dot{\xi} x \partial_{x}-2\left(\delta_{1}\left(-i \partial_{x}\right)+\left(V_{1} \xi-\dot{\delta_{1}}\right) x\right)\right] \\
& =\frac{1}{2}\left\{\left(\frac{2 a}{\xi}\left(1+\frac{1}{4} \dot{\xi}^{2}\right)+\dot{a} \dot{\xi}\right) x-(a \dot{\xi}+2 \dot{a} \xi)\left(-i \partial_{x}\right)-2 a\left(V_{1} \xi-\dot{\delta_{1}}\right)+2 \dot{a} \delta_{1}\right\}
\end{aligned}
$$

to be compared with the infinitesimal change of $\mathcal{E} \mathcal{L}$ under the transformation $x \rightarrow$ $x+\varepsilon a, \delta_{1} \rightarrow \delta_{1}+\varepsilon\left(\xi \dot{a}-\frac{1}{2} a \dot{\xi}\right), \delta_{2} \rightarrow \delta_{2}+\varepsilon\left(\left(\delta_{1} \dot{a}-a \dot{\delta_{1}}\right)+\xi \dot{b}\right), V_{1} \rightarrow V_{1}-2 \varepsilon\left(\ddot{a}+V_{2} a\right)$. This is a straightforward computation, which requires the use of the equation defining $\xi$, namely, $\ddot{\xi}=\frac{1}{\xi}\left(1+\frac{1}{4} \dot{\xi}^{2}\right)-2 V_{2} \xi$.

Using the parametrization of $\mathcal{S}_{\leq 2 \text {,gen }}^{\text {aff }}$ by the vector invariant $\left(\xi, \delta_{1}, \delta_{2}\right)$, one can easily define a natural symplectic structure on a linear space $\Omega$ and a Hamiltonian action of SV on $\Omega$ reproducing the SV-action on $\mathcal{S}_{\leq 2, \text { gen }}^{\text {aff }}$.

Definition 4.5. Let $\Omega \simeq C^{\infty}\left(\mathbb{R} / 2 \pi \mathbb{Z}, \mathbb{R}^{4}\right)$ be the linear manifold consisting of all $2 \pi$-periodic vector-valued $C^{\infty}$ functions $\boldsymbol{X}(\tau):=(p, q, E, t)(\tau), \tau \in \mathbb{R} / 2 \pi \mathbb{Z}$ with singular Poisson structure defined by

$$
\left\{p(\tau), q\left(\tau^{\prime}\right)\right\}=\delta\left(\tau-\tau^{\prime}\right), \quad\left\{E(\tau), t\left(\tau^{\prime}\right)\right\}=\delta\left(\tau-\tau^{\prime}\right)
$$

See for instance [14], Chap. X for some remarks on distribution-valued singular Poisson structures on infinite-dimensional spaces. The energy $E$ is canonically conjugate to $t$, which allows us to consider generalized canonical transformations for which $t$ is a coordinate. This usual trick for Hamiltonian systems with timedependent Hamiltonians can for instance be found in [9]. Hamiltonian vector fields $\mathcal{X}_{H}$, for $H=H(p, q, E, t)$, acts separately on each fiber $\tau=$ constant, namely,

$$
\left(\mathcal{X}_{H} f\right)(\tau):=\left\{\left(\partial_{p} H \partial_{q}-\partial_{q} H \partial_{p}+\partial_{E} H \partial_{p}-\partial_{t} H \partial_{E}\right) f\right\}(\tau) .
$$

Definition 4.6. Let $\left(\xi, \delta_{1}, \delta_{2}\right)$ be a triple of $2 \pi$-periodic functions. Define $\Phi:=$ $\Phi\left(\xi, \delta_{1}, \delta_{2}\right)$ to be the following functional on $\Omega$,

$$
\begin{aligned}
\langle\Phi, \boldsymbol{X}\rangle= & \oint\left\{\xi(t(\tau)) E(\tau)+\frac{1}{2} \dot{\xi}(t(\tau)) p(\tau) q(\tau)+\delta_{1}(t(\tau)) p(\tau)\right. \\
& \left.-\dot{\delta}_{1}(t(\tau)) q(\tau)+\delta_{2}(t(\tau))\right\} d \tau
\end{aligned}
$$


Theorem 4.7. Represent $\mathcal{L}_{f}+\mathcal{Y}_{g}+\mathcal{M}_{h} \in \mathfrak{s v}$ by the Hamiltonian vector field $\mathcal{X}_{H(f, g, h)}$ associated to

$$
H(f, g, h):=-\left(f(t) E+\frac{1}{2} \dot{f}(t) p q+\frac{1}{4} \ddot{f} q^{2}\right)-(g(t) p+\dot{g}(t) q)-h(t) .
$$

Then the action of $\mathcal{X}_{H}$ on the functional $\Phi\left(\xi, \delta_{1}, \delta_{2}\right)$ coincides with that given in Theorem 4.4.

Proof. Observe that the map from $\mathfrak{s v}$ to the Lie algebra of vector fields on $\Omega$ given by $\mathcal{L}_{f}+\mathcal{Y}_{g}+\mathcal{M}_{h} \rightarrow \mathcal{X}_{H(f, g, h)}$ is a Lie algebra homomorphism. The vector field $\mathcal{X}_{H}$ is given explicitly by

$$
\begin{aligned}
\mathcal{X}_{H(f, g, h)}= & -\left[\frac{1}{2} \dot{f}(t)\left(q \partial_{q}-p \partial_{p}\right)+f(t) \partial_{t}-\frac{1}{2} \ddot{f}(t) q \partial_{p}\right]-\left[g(t) \partial_{q}-\dot{g}(t) \partial_{p}\right] \\
& +\left[\left(\frac{1}{2} \ddot{f}(t) p q+\dot{f}(t) E+\frac{1}{4} f^{\prime \prime \prime}(t) q^{2}\right)+(\dot{g} p+\ddot{g} q)+\dot{h}(t)\right] \partial_{E} .
\end{aligned}
$$

The rest is a straightforward computation.

Let us conclude this section by computing the monodromy for "resonant" operators of types (i)bis and (iii)bis.

Consider any resonant operator $D$. The associated classical monodromy is unipotent. We choose $\xi \in \operatorname{Stab}_{V_{2}}$ to be purely imaginary, $\xi:=i \eta$ as before (see Sec. 3.2). A generalized Ermarkov-Lewis invariant may then be defined as

$$
\mathcal{E} \mathcal{L}(D)=\frac{1}{2 \xi}\left[\left(i \xi \partial_{x}+\frac{1}{2} \dot{\xi} x\right)^{2}\right]+\frac{i}{2}\left[d\left(-i \partial_{x}\right)+e x+f\right],
$$

where $d, e, f$ are defined as in Theorem 4.4 but with $\xi$ replaced by $\eta$ (see Eq. (4.10) for notations). Hence

$$
\frac{\mathcal{E} \mathcal{L}(D)-i k}{\xi}=-\frac{1}{2}\left[\left(\partial_{x}-\frac{i}{2} \frac{\dot{\eta}}{\eta} x\right)^{2}-\frac{d}{\eta}\left(-i \partial_{x}\right)-\frac{e}{\eta} x-\frac{f}{\eta}\right]-\frac{k}{\eta} .
$$

Suppose $\mathcal{E} \mathcal{L}(D) \psi_{k}=i k \psi_{k}$ and set

$$
\tilde{\psi}_{k}=\exp \left(-\frac{i}{4} \frac{\dot{\eta}}{\eta} x^{2}+\frac{i}{2} \frac{d}{\eta} x\right) \psi_{k}
$$

Then a simple calculation gives

$$
\left[\partial_{x}^{2}-\left(\frac{1}{2} d \frac{\dot{\eta}}{\eta^{2}}+\frac{e}{\eta}\right) x+\frac{-f+2 k}{\eta}+\frac{1}{4}\left(\frac{d}{\eta}\right)^{2}\right] \tilde{\psi}_{k}=0 .
$$

If $D$ is of type (i)bis, then $d, e, f$ (easy to obtain from Theorem 4.4 and the isotropy algebra given in Sec. 2) satisfy $\frac{1}{2} d \frac{\dot{\eta}}{\eta^{2}}+\frac{e}{\eta}=0$ identically, so the model operator is (up to a constant) the Laplacian as for case (iii). Then the monodromy 
can be computed along the same lines as in Sec. 3.6, with a time-independent shift in $k$ due to the function $-f+\frac{1}{4} \frac{d^{2}}{\eta}=\frac{3 C^{2}}{128 n^{2}}$.

Lemma 4.8. Let $D=-2 i \partial_{\theta}-\partial_{x}^{2}+n^{2} x^{2}+C \cos n(\theta-\sigma / 2) x+\gamma$ be a Schrödinger operator of type (i)bis. Set

$$
\psi_{k, \pm}(\theta, x)=e^{\frac{i}{4} \frac{\dot{\eta}}{\eta} x^{2}-\frac{i}{2} \frac{d}{\eta} x} \cdot e^{ \pm i \sqrt{\frac{2 k^{\prime}}{\eta}} x},
$$

with $d=-\frac{C}{4 n} \sin 3 n(\theta-\sigma / 2), \eta=1-\cos (2 n \theta-\sigma), k^{\prime}=k+3\left(\frac{C}{16 n}\right)^{2}$. Then

$$
D \psi_{k, \pm}=\left(\frac{2 k^{\prime}}{\eta}+\frac{1}{4}\left(\frac{d}{\eta}\right)^{2} \mp d \eta^{-3 / 2} \sqrt{2 k^{\prime}}-\frac{i}{2} \frac{\dot{\eta}}{\eta}+\gamma\right) \psi_{k, \pm} .
$$

Proof. Tedious computations.

Apart from the time-periodic shift $\frac{1}{4}\left(\frac{d}{\eta}\right)^{2}=\left(\frac{C}{16 n}\right)^{2} \frac{\sin ^{2} 3 n(\theta-\sigma / 2)}{\sin ^{4} n(\theta-\sigma / 2)}$ (which is integrable on the contour $\Gamma$ ) and the time-independent shift in $k$, one is left once again with a phase proportional to $k / \eta$ (note that the term in $d \eta^{-3 / 2} \sqrt{2 k^{\prime}}$ is irrelevant since $\int_{0}^{2 \pi}\left(d \eta^{-3 / 2}\right)(\theta) d \theta=0$ by Lemma 4.3 ; recall $d=-2 \delta_{1}$ by Theorem 4.4$)$.

Hence one obtains:

Theorem 4.9. Let $\psi(0) \in L^{2}(\mathbb{R})$, with decomposition

$$
\psi(0, x):=\int_{\mathbb{R}_{+}} \bar{c}_{+}(k) \psi_{k,+}(0, x) d k+\int_{\mathbb{R}_{+}} \bar{c}_{-}(k) \psi_{k,-}(0, x) d k .
$$

Then the solution of the type (i)bis Schrödinger equation

$$
\left(-2 i \partial_{\theta}+\partial_{x}^{2}+n^{2} x^{2}+C \cos (n \theta-\sigma / 2) \cdot x+\gamma\right) \psi=0
$$

with initial state $\psi(0)$ is given at time $\theta=2 \pi$ by

$$
\psi(2 \pi, x)=\int_{\mathbb{R}_{+}} \bar{c}_{+}(k) e^{k^{\prime} T-i \pi \tilde{\gamma}} \psi_{k,+}(0, x) d k+\int_{\mathbb{R}_{+}} \bar{c}_{-}(k) e^{k^{\prime} T-i \pi \tilde{\gamma}} \psi_{k,-}(0, x) d k,
$$

where $k^{\prime}=k+3\left(\frac{C}{16 n}\right)^{2}, T=\int_{0}^{2 \pi} \frac{d u}{\xi(u)}(T$ is purely imaginary) and

$$
\tilde{\gamma}=\gamma+\frac{1}{4} \int_{\Gamma}\left(\frac{d}{\eta}\right)^{2}(\theta) d \theta
$$

The associated monodromy operator in $\mathcal{B}\left(L^{2}(\mathbb{R}), L^{2}(\mathbb{R})\right)$ is unitarily equivalent to the unitary operator on $L^{2}\left(\mathbb{R}_{+}\right)$given by the multiplication by the function $k \rightarrow$ $e^{k T-i \pi \tilde{\gamma}}$.

Suppose now $D$ is of type (iii)bis. Then the $x$-coefficient in the transformed Ermakov-Lewis operator (4.25) does not vanish, so one must take for "model operator" $-\partial_{x}^{2}+x$, whose eigenfunctions are related to the Airy function. The solution of the monodromy problem will be given by a series of lemmas. In the sequel, 
$\eta=(1+\sin n \theta)(1+\alpha \sin n \theta)$ is the (real-valued and non-negative) invariant, and $\eta^{1 / 2}=(1+\alpha \sin n \theta)^{1 / 2} \cos \left(\frac{\pi}{4}-n \frac{\theta}{2}\right) x$ is the smooth square-root of $\eta$ chosen in Sec. 2.

Lemma 4.10. Let Ai be the entire function, solution of the Airy differential equation $\left(-\partial_{x}^{2}+x\right) A i(x)=0$, defined on the real line as

$$
A i(x)=\frac{1}{\pi} \int_{0}^{\infty} \cos \left(\frac{t^{3}}{3}+x t\right) d t .
$$

It is (up to a constant) the only solution of the Airy differential equation which do not increase exponentially on $\mathbb{R}_{+}$. The functions $f_{k}(x):=A i(x-k), k \in \mathbb{R}$ define (up to a coefficient) a complete orthonormal system of generalized eigenfunctions of the self-adjoint closure of the Airy operator $-\partial_{x}^{2}+x$ with core $C_{0}^{\infty}(\mathbb{R}) \subset L^{2}(\mathbb{R})$.

Proof. It is easy by using a Fourier transform.

Lemma 4.11. The $x$-coefficient in the transformed Ermakov-Lewis invariant (4.25) reads

$$
\frac{1}{2} d \frac{\dot{\eta}}{\eta^{2}}+\frac{e}{\eta}=-C_{\alpha} \eta^{-3 / 2}
$$

where $C_{\alpha}=(1-\alpha)(1+\alpha / 2) \sqrt{1-\alpha^{2}}$.

Proof. Computations similar to that of Lemma 4.3 (with the simple difference that $\xi \ddot{\xi}-\frac{1}{2} \dot{\xi}^{2}+2 V_{2} \xi^{2}=0$ here) yield

$$
\frac{d}{d \theta}\left(\eta^{-\frac{1}{2}} d\right)=\eta-\frac{C_{\alpha}}{\eta}
$$

where $C_{\alpha}$ is some constant which must be chosen in order that the right-hand side be $2 \pi$-periodic. Note that the singularities in the above equation are only apparent; one may avoid them altogether by using a contour $\Gamma$ in the upper-half plane as in Sec. 3.2. Since $\int_{0}^{2 \pi} \eta=2 \pi(1+\alpha / 2)$ and $\int_{\Gamma} \frac{d \theta^{\prime}}{\eta\left(\theta^{\prime}\right)}=-\frac{2 \pi}{(1-\alpha) \sqrt{1-\alpha^{2}}}$ (see Proposition 2.2.3), this gives $C_{\alpha}=(1-\alpha)(1+\alpha / 2) \sqrt{1-\alpha^{2}}$. Then a straightforward computation yields formula (4.32).

Lemma 4.12. Set

$$
\begin{aligned}
\psi_{k}(\theta, x)= & \exp \left(\frac{i}{4} \frac{\dot{\eta}}{\eta} x^{2}-\frac{i}{2} \frac{d}{\eta} x\right) \cdot \eta^{-\frac{1}{2}} A i \\
& \times\left(x C_{\alpha}^{1 / 3} \eta^{-\frac{1}{2}}-C_{\alpha}^{-2 / 3}\left(-f+2 k+\frac{1}{4} \frac{d^{2}}{\eta}\right)\right) .
\end{aligned}
$$

Then

$$
D \psi_{k}(\theta, x)=\left(\frac{2 k}{\eta}+\left(\frac{i}{2} \frac{\dot{\eta}}{\eta}+\frac{1}{2}\left(\frac{d}{\eta}\right)^{2}-\frac{f}{\eta}\right)\right) \psi_{k}(\theta, x)
$$


Proof. The $\psi_{k}$ are obtained as in Sec. 3.5 (monodromy of hyperbolic operators) by taking a complete orthonormal system of generalized eigenfunctions $\tilde{\psi}_{k}$ for the transformed Ermakov-Lewis invariant (4.25) and going back to the functions $\psi_{k}$. Then (4.35) is proved by a direct tedious computation.

One may now conclude:

Lemma 4.13. Let $\psi(0) \in L^{2}(\mathbb{R})$, with decomposition

$$
\psi(0, x):=\int_{\mathbb{R}} \bar{c}(k) \psi_{k}(0, x) d k .
$$

Then the solution of the type (iii)bis Schrödinger equation

$$
\left(-2 i \partial_{\theta}+\partial_{x}^{2}+v_{n, \alpha} x^{2}+C(1+\alpha \sin n \theta)^{1 / 2} \cos \left(\frac{\pi}{4}-n \frac{\theta}{2}\right) \cdot x+\gamma\right) \psi=0
$$

with initial state $\psi(0)$ is given at time $\theta=2 \pi$ by

$$
\psi(2 \pi, x)=\int_{\mathbb{R}} \bar{c}(k) e^{k T-i \pi \tilde{\gamma}} \psi_{k}(0, x) d k,
$$

where $T=\int_{0}^{2 \pi} \frac{d u}{\xi(u)}(T$ is purely imaginary $)$ and

$$
\tilde{\gamma}=\gamma+\int_{\Gamma}\left(-\frac{f}{\eta}+\frac{1}{2}\left(\frac{d}{\eta}\right)^{2}\right)(\theta) d \theta .
$$

The associated monodromy operator in $\mathcal{B}\left(L^{2}(\mathbb{R}), L^{2}(\mathbb{R})\right)$ is unitarily equivalent to the unitary operator on $L^{2}(\mathbb{R})$ given by the multiplication by the function $k \rightarrow$ $e^{k T-i \pi \tilde{\gamma}}$.

\section{Acknowledgment}

We wish to thank A. Joye for a useful discussion in Cergy.

\section{References}

1. M. Abramowitz, A. Stegun, M. Danos and J. Rafelski, Handbook of Mathematical Functions (Harri Deutsch, 1984).

2. J. Balog, L. Fehér and L. Palla, Coadjoint orbits of the Virasoro algebra and the global Liouville equation, Int. J. Mod. Phys. A 13 (1998) 315-362.

3. F. A. Berezin and M. A. Shubin, The Schrödinger Equation (Kluwer, 1991).

4. A. Bohm, A. Mostafazadeh, H. Koizumi, Q. Niu and J. Zwanziger, The Geometric Phase in Quantum Systems, Texts and Monographs in Physics (Springer, 2003).

5. S. Bouquet and H. R. Lewis, A second invariant for one-degree-of-freedom, timedependent Hamiltonians given a first invariant, J. Math. Phys. 37 (1996) 55095517.

6. S. Bouquet, M. Feix, P. G. L. Leach and H. R. Lewis, Representations of onedimensional Hamiltonians in terms of their invariants, J. Math. Phys. 33 (1992) $591-598$. 
7. A. Erdelyi, W. Magnus, F. Oberhettinger, F. Tricomi and H. Bateman, Higher Transcendental Functions (McGraw-Hill, 1953).

8. I. M. Gelfand and G. E. Shilov, Generalized Functions, Vol. 1 (Academic Press, 1964).

9. H. Goldstein, Classical Mechanics (Addison-Wesley, 1959).

10. I. S. Gradshteyn and I. M. Ryzhik, Table of Integrals, Series, and Products (Academic Press, 1980).

11. M. F. Guasti and H. Moya-Cessa, Solution of the Schrödinger equation for timedependent 1D harmonic oscillators using the orthogonal functions invariant, J. Phys. A 36 (2003) 2069-2076.

12. M. F. Guasti and H. Moya-Cessa, Coherent states for the time-dependent harmonic oscillator: The step function, Phys. Lett. A 311 (2003) 1-5.

13. L. Guieu, Sur la géométrie des orbites de la représentation coadjointe du groupe de Bott-Virasoro, PhD thesis, Université Aix-Marseille I, 1994.

14. L. Guieu and C. Roger, L'Algèbre et le Groupe de Virasoro: Aspects Géométriques et Algébriques, Généralisations (CRM, 2007).

15. V. Guillemin and S. Sternberg, Symplectic Techniques in Physics (Cambridge Univ. Press, 1984).

16. G. Hagedorn, Raising and lowering operators for semiclassical wave packets, Ann. Phys. 269 (1998) 77-104.

17. G. Hagedorn, M. Loss and J. Slawny, Non-stochasticity of time-dependent quadratic Hamiltonians and the spectra of canonical transformations, J. Phys. A 19 (1986) 521-531.

18. M. Henkel, Schrödinger invariance and strongly anisotropic critical systems, J. Stat. Phys. 75 (1994) 1023.

19. M. Henkel, Phenomenology of local scale invariance: From conformal invariance to dynamical scaling, Nucl. Phys. B 641 (2002) 405.

20. M. Henkel, Phase-ordering kinetics: Ageing and local scale-invariance, cond-mat/ 0503739 .

21. A. Joye, Geometric and mathematical aspects of the adiabatic theorem of quantum mechanics, PhD thesis, Ecole Polytechnique Fédérale de Lausanne (1992).

22. B. Khesin and R. Wendt, The Geometry of Infinite-Dimensional Lie Groups, Series of Modern Surveys in Mathematics, Vol. 51 (Springer, 2008).

23. A. A. Kirillov, Infinite-dimensional Lie groups: Their orbits, invariants and representations, The Geometry of Moments, Lecture Notes in Math., Vol. 970 (Springer, 1982), pp. 101-123.

24. V. F. Lazutkin and T. F. Pankratova, Normal forms and versal deformations for Hill's equation, Funct. Anal. Appl. 9 (1975) 306-311.

25. P. G. L. Leach and H. R. Lewis, A direct approach to finding exact invariants for one-dimensional time-dependent classical Hamiltonians, J. Math. Phys. 23 (1982) 2371-2374.

26. H. R. Lewis and W. B. Riesenfeld, An exact quantum theory of the time-dependent harmonic oscillator and of a charged particle in a time-dependent electromagnetic field, J. Math. Phys. 10 (1969) 1458-1473.

27. W. Magnus and S. Winkler, Hill's Equation (John Wiley and Sons, 1966).

28. U. Niederer, The maximal kinematical invariance groups of Schrödinger equations with arbitrary potentials, Helv. Phys. Act. 47 (1974) 167-172.

29. P. B. E. Padilla, Ermakov-Lewis dynamic invariants with some applications, Master Thesis, Institutot de Fisica (Guanajuato, Mexico), available on arXiv:math-ph/ 0002005. 
30. J. R. Ray and J. L. Reid, Invariants for forced time-dependent oscillators and generalizations, Phys. Rev. A 26 (1982) 1042-1047.

31. C. Roger and J. Unterberger, The Schrödinger-Virasoro Lie group and algebra: Representation theory and cohomological study, Ann. Henri Poincaré 7 (2006) 1477-1529.

32. M. Henkel and J. Unterberger, Schrödinger invariance and space-time symmetries, Nucl. Phys. B 660 (2003) 407. 